\title{
Geometric Approach to Measure-Based Metric in Image Segmentation
}

\author{
Vladimir Kluzner · Gershon Wolansky • Yehoshua Y. Zeevi
}

Published online: 17 October 2008

(C) Springer Science+Business Media, LLC 2008

\begin{abstract}
The Mumford-Shah functional and related algorithms for image segmentation involve a tradeoff between a two-dimensional image structure and one-dimensional parametric curves (contours) that surround objects or distinct regions in the image.

We propose an alternative functional that is independent of parameterization; it is a geometric functional given in terms of the surfaces representing the data and image in the feature space. The $\Gamma$-convergence technique is combined with the minimal surfaces theory to yield a global generalization of the Mumford-Shah segmentation function.
\end{abstract}

Keywords Image segmentation - Measure-based metric . Geometric functional · Gamma-convergence $\cdot$ Minimal surfaces

\section{Introduction}

Let $g(x, y)$ be the intensity of the light signal impinging on a planar image domain $B$ at a point $(x, y)$. The image $g(x, y)$ is expected to be discontinuous along the edges of the objects. The definition of the segmentation depends on whether one approaches the problem at the level of the image as a

\author{
V. Kluzner $(\bowtie)$ \\ IBM Haifa Research Lab, IBM Corporation, Haifa University, \\ Mount Carmel, Haifa, 31905, Israel \\ e-mail:kvladi@il.ibm.com \\ G. Wolansky · Y.Y. Zeevi \\ Technion City, Haifa 32000, Israel \\ G. Wolansky \\ e-mail: gershonw@math.technion.ac.il \\ Y.Y. Zeevi \\ e-mail: zeevi@ee.technion.ac.il
}

whole or, alternatively, considers the image as a collection of edge fragments. In the first case it is natural to consider the partitioning of the image into smaller structures. In the second case it becomes more natural to consider the problem of grouping of elements into larger structures. In both cases, the following questions arise: (i) What exactly is the goal of the segmentation process? (ii) Is segmentation feasible? These questions are important for understanding of the above process. Without a clear conception of the task and its requirements no satisfactory progress in this area can be made.

The above dichotomy into local vs. global, and related heuristic approaches, were later circumvented by the variational approach to segmentation, adopted by us and further developed in this paper.

Morel and Solimini [35] showed that any heuristic segmentation method may be translated into a variational one. Variational formulations summarize all criteria concerning a set of edges $K$ in a single real-valued functional $F(K)$. For any set of edges or "segmentation" $K$ there is an associated value $F(K)$ which states the merit of this segmentation. In general, this functional is a trade off between fidelity and the number of the segments involved. This leads to an obvious comparison principle between any pair of possible segmentations.

The Mumford-Shah variational model [36] turned into a general framework for image segmentation. It defined the segmentation problem as a joint smoothing/edge detection problem as follows: given an image $g$, it seeks simultaneously a set $K$ of discontinuities, the "edges" of $g$, decomposing the planar domain $B$ ("pixel space") to disjoint connected open subsets $B_{i}, i=1 \ldots n$, so that $B=B_{i} \cup \ldots \cup$ $B_{n} \cup K$, and a function $u$ differentiable on $\cup B_{i}$, which is allowed to be discontinuous across $K$. Morel and Solimini [35] showed that many other segmentation algorithms 
are particular cases of Mumford-Shah functional or closely related to it. Unfortunately, minimizing the Mumford-Shah functional is a very difficult task because of the subtle interaction of the two-dimensional term $u$ and the onedimensional term $|K|$. It isn't also obvious that the minimizing problem is well posed.

There are also dynamic models which use the local information along the deforming counters. The "snakes" model, introduced by Kass, Witkin and Terzopoulos [24], provided an accurate localization of edges near a given initialization of the curve. Caselles et al. [8] introduced a geometric flow segmentation model, overcoming successfully problems characteristic of snake method. A unification of both the parametric and the level set geometric model, known as the geodesic active contours, was suggested by Caselles et al. [9]. In order to accelerate the motion of the geodesic active contours, Goldenberg et al. [20] proposed to add an additional force that comes from area minimization term and motivated by the balloon force [16]. An attempt to merge the active contours technique and Mumford-Shah functional theory was made by Chan and Vese [14], who proposed a minimal variance criterion (edgeless active contours). Chan, Sandberg and Vese [13] proposed an extension to the vector case, where the model can detect edges present in at least one of the channels and not necessarily in all channels. The geodesic contours model and edgeless active contours model were integrated into a unified algorithm in the work of Kimmel [25]. At first, the arclength term in [14] was replaced by the more general weighted arclength term, also known in the geodesic active contour functional [9] as a data sensitive regularization term. This yielded better results in most cases and simplified the regularization used by Chan and Vese [14]. Second, the alignment term as part of other driving forces of an active contour was added. The above combined approach which accounts for both the gradients between regions and region's homogeneity, may produce better results for various images.

The geometric framework developed by Sochen et al. in [44] is conceptually close to ours. They introduced the set of tools for enhancing while preserving either the multichannel edges or their texture features. The images are treated as manifolds (surfaces in the $2 D$ case) embedded in a higher dimensional space-feature manifold. The manifold metric is a free "parameter" of the framework. For the induced metric the norm becomes simply the area or the volume of the image manifold, and the flow is towards minimal surface solution. Minimizing the area over the space of mappings, Sochen et al. in [45] obtained the area minimization direction given by applying the second order differential operator of Beltrami on the feature coordinates, which in fact is a generalization of the Laplacian associated with flat spaces. In fact, for the grey level case the above operator describes the mean curvature flow of the image surface divided by the determinant of induced metric. The extension of the above approach for non-Euclidean embedding space was treated by Sochen and Zeevi in [46]. Another example of induced metric may be taken from [42]: Sagiv, Sochen and Zeevi proposed a novel approach for texture segmentation, which enables the use the geodesic active contours mechanism in the Gabor spatial feature space of images, by generalizing the edge indicator function. Following the model [8] developed for grey level images, Sagiv, Sochen and Zeevi [43] generalized it to texture segmentation model. The energy functional to be minimized can be seen as a natural extension of the functional in [25], where the term which accounts for the arc-length of the curve is replaced by the geodesic length of the curve, weighted by the gradient information.

A novel and powerful approach has emerged from the mathematical theory of approximation of functionals via $\Gamma$-convergence. The idea was to approximate the functional by a different, parameter-dependent, functional that is expected to be more regular. As a result, the minimizer of approximating functional should approximate the minimizer of original one, while enjoying greater regularity. The above technique, merged with the Beltrami framework [44] was originally presented in [27] as two different approaches possible in the treatment of the segmentation function. First, the edge indicator function $E$ was defined on the image surface manifold. Here the Polyakov action [39] was used as an adaptive smoothing metric for both the color coordinates and the segmenting function. In the second approach the edge indicator function was defined over the image plane. The metric was as before, the induced metric, but this time the Polyakov action was used only for the feature coordinates. In the above work the generalized $\Gamma$ convergence technique was applied to Mumford-Shah functional with reference to two aspects: multi-channel images were analyzed and the $\mathbb{L}_{2}$ norm in the smoothness term was replaced by the Polyakov action. Eventually, in [4] and [5] the Mumford-Shah functional was generalized to color images, for segmentation and restoration purposes respectively, using the "image as a manifold" interpretation, while the length term remained the same.

We propose an alternative to the Mumford-Shah functional (Sect. 2). The concept behind this alternative is a geometric functional which is independent of parameterization, and can be implemented in terms of the geometry of surfaces representing the data and image in the feature space. Starting with the original version of the MumfordShah segmentation functional given in [36] and using the theory of minimal surfaces, we bring the full construction of our functional. The minimization problem for relaxed functional with parametric representation is treated (Sect. 3) and the detailed solution of the minimization problem for the relaxed functional with non-parametric representation is presented (Sect. 4). 


\section{Measure-Based Metric Function for the} Segmentation Functional

\subsection{Preliminary Analysis}

Mumford and Shah [36] proposed the functional

$$
F_{\mathrm{MS}}(u, K)=\int_{B \backslash K}|\nabla u|^{2}+|K|+\alpha \int_{B}|u-g|^{2},
$$

where $K \subset B$ is a one dimensional set which represents the edges, and $|K|$ is the length of this set (understood as the one-dimensional Hausdorff measure of $K$ ). A minimizer $\{u, K\}$ of $F_{\mathrm{MS}}$ thus produces the required image $u$ as well as the edges $K$.

We propose an alternative functional. The concept behind this alternative is an object which is independent of parameterization, i.e a geometric functional which may be given in terms of the geometry of surfaces representing the data and image in the feature space (see below). Considering the image $u$ as a two-dimensional surface, we shall replace the first two terms of (2.1) by the area of this surface. This allows sharp discontinuities (edges) of the image in the form of surface folding. This idea is not new (see, e.g., [44]). However, to make the third term of (2.1) fit the geometrical description, we must replace it by another metric $\mathcal{D}(g, u)$ representing the distance between the two surfaces.

To motivate this change, we point out that the term $\int|u-g|^{2}$ connects locally the image $u(b)$ for each $b \in B$ to the data $g$ at the same point $b$. Thus, this term implicitly assumes that there is a one-to-one correspondence between points of the data to points of the image. It is well known that this is never the case, since defocusing and mapping errors imply that each pixel point is influenced by a domain rather than by a single point of the data.

Moreover, the proposed change allows us to replace the deterministic data $g$ by a random one. For this we replace $g(b)$ by a positive measure $\mu_{b}(d y) d b$ where $y$ is a parameterization of the feature fiber (see below). Further, to different pixels there may correspond different amount of data, so $\int \mu_{b}(d y) \leq 1$ for each pixel $b$ where strong inequality and even zero value may not be excluded for some of the pixels.

Our objective is to define a distance $\mathcal{D}(\mu, U)$ between the data measure $\mu$ and the image surface $U$.

Before proceeding, let us define the feature space. Let $\mathbf{Y}$ be a set representing the possible data at a single pixel $b \in B$. It may be considered as a real number (the brightness) or a vector (if several color channels are present or, for example, a Gabor-wavelet filter, see [26]). We shall assume here that $\mathbf{Y}=\mathbb{R}^{m}$. The feature space is, then, defined as the cylinder $E:=B \times \mathbf{Y} \subset \mathbb{R}^{m+2}$. The data $g$ is represented as a measure $\mu$ supported in $E$.

To define an image $u$, let us consider the two-dimensional unit disc $S:\{|s|<1\}$ diffeomorphic to $B$. We represent the image as a mapping $U: S \rightarrow E$ such that $U(\partial S) \subset \partial B \times \mathbf{Y}$ is projected onto $\partial B$. Coordinates in the feature space are described by $z:=\{b, y\}$.

The feature space $E$ is endowed with a metric

$d z^{2}=\gamma d b^{2}+d y^{2}:=\sum_{i, j=1}^{m+2} h_{i, j} d z_{i} d z_{j}$,

where $d b^{2}=d b_{1}^{2}+d b_{2}^{2}, d y^{2}=\sum_{i=1}^{m} d y_{i}^{2}$ are the Euclidean metric in $\mathbb{R}^{2}$ and $\mathbb{R}^{m}$, respectively, while $\gamma>0$ represents the relation between the geometric (pixel-domain) and feature metrics. Here

$h_{i, j}=\left\{\begin{array}{ll}\gamma, & 1 \leq i=j \leq 2 \\ 1, & 3 \leq i=j \leq m+2 . \\ 0, & i \neq j\end{array}\right.$.

With this setting, the embedding $U(S)$ into $E$ is endowed with the induced metric

$\Gamma_{i, j}(U)=U_{s_{i}}^{T} h U_{s_{j}} \quad$ for $i, j=1,2$.

Setting $|\Gamma(U)|:=\left|\Gamma_{1,1}(U) \Gamma_{2,2}(U)-\Gamma_{1,2}^{2}(U)\right|^{1 / 2}$, the surface area of $U(S)$ is

$A(U)=\int_{S}|\Gamma(U)(s)| d s$.

Let us now replace the first two terms of (2.1) by (2.2), and the last term by a distance $\mathcal{D}(\mu, U)$ between the embedded surface $U(S)$ and the data measure $\mu$ :

$F(U)=A(U)+\alpha \mathcal{D}^{2}(\mu, U)$.

By our convention, the distance $\mathcal{D}$ should only depend on the image $U(S)$ and not on a particular parameterization. With this assumption, we replace $A(U)$ by the quadratic form

$\widetilde{A}(U)=\frac{1}{2} \int_{S} \operatorname{Tr}\left(\nabla U(s)^{T} h \nabla U(s)\right) d s$,

where $\operatorname{Tr}(\cdot)$ is a trace of a given matrix. Note that

$\widetilde{A}(U) \geq A(U)$,

which reduces to an equality if the parameterization of $U$ is conformal, namely

$\Gamma_{1,1}(U)=\Gamma_{2,2}(U), \quad \Gamma_{1,2}(U)=\Gamma_{2,1}(U)=0$.

We replace $F(U)$ by

$\widetilde{F}(U)=\widetilde{A}(U)+\alpha \mathcal{D}^{2}(\mu, U)$

and obtain by (2.5)

$\widetilde{F}(U) \geq F(U)$, 
and equality if the embedded $U$ is a conformal mapping from $S$ to $E$.

For parametric representation of an image used in this section we cannot exclude the possibility that the optimal image is folded over the pixel space $B$. If this is the case, then some pixels of the image over $B$ may have multiple values.

In Sect. 2.2 we introduce the distance function $D(\mu, v)$ and the associated metric $\mathcal{D}(\mu, U)$. The proposed definition of $\mathcal{D}$ has the following advantages: (i) it is lower semicontinuous in a proper topology, thus guarantees the existence of a minimizer, (ii) it can be relaxed into a form which is convenient for numerical implementation. In Sect. 2.3 we study the functionals $F$ and $\widetilde{F}$ over suitable domains. In particular, we show that a minimizer of $\widetilde{F}$ exists and is a conformal mapping, hence it is also a minimizer of $F$. In Sect. 2.4 we introduce the relaxation of $D$ into $D_{\beta}$ for $\beta>0$ such that $\lim _{\beta \rightarrow 0} D_{\beta}=D$ is in the sense of $\Gamma$-convergence. This, in particular, will enable us in Sect. 2.5 to relax $\widetilde{F}$ into $\widetilde{F}_{\beta}$, whose $\Gamma$-limit at $\beta \rightarrow 0$ coincides with $\widetilde{F}$.

\subsection{The Distance Function and Parameter Dependent Metric}

Recall the Hausdorff distance $d_{H}$ between two closed sets $A, B \subset E$

$d_{H}(A, B)=\sqrt{\sup _{v \in B} \inf _{u \in A}|u-v|^{2}}+\sqrt{\sup _{u \in A} \inf _{v \in B}|u-v|^{2}}$.

It is tempting to define the distance between a pair of measures by

$D(\mu, v)=d_{H}(\operatorname{supp}(\mu), \operatorname{supp}(v))$.

However, it turns out that, by this definition, $D$ is not $C^{*}$ lower semi-continuous with respect to $v$ in the weak topology of measures. So we define

$$
d(A, B)=\sqrt{\sup _{v \in B} \inf _{u \in A}|u-v|^{2}}
$$

and

$$
D(\mu, \nu)=d(\operatorname{supp}(\mu), \operatorname{supp}(\nu)) .
$$

Note that $D(\mu, v)=0$, if $\operatorname{supp}(v) \subseteq \operatorname{supp}(\mu)$. We shall later consider the measure $\mu$ as representing the data, while $v$ representing the image. In general, the data can be nondeterministic, so $\mu$ could be supported on a set of positive Lebesgue measure in the feature space. On the other hand, the measure $v$ is always supported on a two-dimensional manifold. The condition $\operatorname{supp}(v) \subseteq \operatorname{supp}(\mu)$ represents for us an image that fits the data.

We shall see below that $D$ is $C^{*}$ lower semi-continuous with respect to $v$ (but not with respect to $\mu$ ). Indeed, the data $\mu$ is fixed, so we have to care only about the dependence on $v$, which represents the image on which we minimize.

Given a continuous function $f$ on $E$, let

$\sup _{v}(f)=\sup _{v \in \operatorname{supp}(v)} f(v)$.

Similarly

$\inf _{\mu}(f)=\inf _{u \in \operatorname{supp}(\mu)} f(u)$.

To show the next fact, we use the Portmanteau Theorem ([3], Sect. 1.2, Theorem 2.1), which, in our present notation, reads respectively in a following manner:

Theorem 2.1 Let $v_{n}, v$ be the measures in $C^{*}$. The following conditions are equivalent:

- $v_{n} \rightarrow v$ in $C^{*}$.

- $\lim _{n \rightarrow \infty} \int f d v_{n}=\int f d v$ for all bounded, uniformly continuous real $f$.

- $\lim \sup _{n \rightarrow \infty} v_{n}(F) \leq v(F)$ for all closed $F$.

- $\liminf _{n \rightarrow \infty} v_{n}(G) \geq v(G)$ for all open $G$.

For proof see [3].

We now claim:

Lemma 2.1 For fixed, continuous $f$, $\sup _{v}(f)$ is lower semicontinuous under the topology of weak $C^{*}$ convergence.

For proof see Appendix A.1.

Assume from now, that $\mu$ is compactly supported in $E$. Setting $f(v)=\inf _{u \in \operatorname{supp}(\mu)}|u-v|^{2}$, we obtain as a corollary:

Corollary 2.1 For fixed, compactly supported $\mu, D(\mu, \nu)$ is lower semi-continuous with respect to $v$ under the topology of weak $C^{*}$ convergence.

Proof Follows immediately from Lemma 2.1 and the fact that $f(v)$ is continuous.

We now replace the measure $v$ by a mapping $U$. Let $l$ be a measure on the parameter space $S$ (say, the uniform Lebesgue measure). We denote the measure $v$ associated with an embedding $U \in \mathbb{H}^{1}(S, E)$ by $\nu_{U}$ and define it by the pullback

$\nu_{U}(\sigma)=l\left(U^{-1}(\sigma \cap U(S))\right)$

for every Borel measurable set $\sigma \subset E$. In terms of its action on a test function $\phi \in C_{0}(E)$ the above measure is defined in the following way:

$\left\langle v_{U}, \phi\right\rangle=\int_{S} \phi(U(s)) d s$.

Next we establish the useful property of the measure $v_{U}$. 
Proposition 2.1 If $U_{n}$ converges weakly to $U$ in $\mathbb{H}^{1}(S, E)$, then $v_{U_{n}}$ converges weakly in $C^{*}$ to $v_{U}$.

Proof The proposition is equivalent to

$\lim _{n \rightarrow \infty} \int_{S} \phi\left(U_{n}(s)\right) d s=\int_{S} \phi(U(s)) d s$

for any $\phi \in C_{0}(E)$. Any function in $C_{0}(E)$ may be approximated by $C_{0}^{1}(E)$ function, thus it is enough to show (2.12) for $\phi \in C_{0}^{1}(E)$. It is obvious that

$$
\begin{aligned}
& \left|\int_{S} \phi\left(U_{n}(s)\right) d s-\int_{S} \phi(U(s)) d s\right| \\
& \quad \leq \int_{S}\left|\phi\left(U_{n}(s)\right)-\phi(U(s))\right| d s \\
& \quad \leq \max _{E}|\nabla \phi| \int_{S}\left|U_{n}(s)-U(s)\right| d s \\
& \quad \leq \max _{E}|\nabla \phi| \cdot \sqrt{|S|} \cdot \sqrt{\left\|U_{n}-U\right\|_{\mathbb{L}^{2}}} .
\end{aligned}
$$

From the compact embedding of $\mathbb{H}^{1}$ into $\mathbb{L}^{2}$ we obtain that the right-hand part tends to 0 when $n \rightarrow \infty$.

We conclude immediately that (2.11) extends to any $U \in$ $\mathbb{H}^{1}(S, E)$ and define

$\mathcal{D}(\mu, U):=D\left(\mu, v_{U}\right)$.

We now claim:

Corollary 2.2 The metric $\mathcal{D}(\mu, U)$ is weakly lower semicontinuous with respect to $U$ in the $\mathbb{H}^{1}$ topology.

Proof Follows immediately from Corollary 2.1 and Proposition 2.1.

\subsection{Existence of the Minimizer of Functional $\widetilde{F}$}

The natural domain for the functional $\widetilde{F}(2.6)$ is the space $\mathbb{H}^{1}(S, E)$. However, a minimizer of $\widetilde{F}$ over this set is trivial, since for the constant map $U=z_{0}$ with $z_{0} \in \operatorname{supp}(\mu)$ we certainly have $\widetilde{F}(U)=0$. To avoid this, we need to implement a proper boundary condition for $\mathbb{H}^{1}(S, E)$.

By the interpretation of this functional, a natural requirement is that $U$ should be a mapping of $S$ onto a disc-like set in $E$ whose boundary $U\left(C_{S}\right), C_{S}:=\partial S$ is the "frame" of the image, given by a Jordan curve on the cylinder $\mathbf{C}:=\partial B \times \mathbf{Y}$. However, this curve must not be specified a-priori, since we assume no a-priori knowledge on the boundary of this image. Referring to the classical theory of minimal surfaces, this suggests to consider the cylinder $\mathbf{C}$ as a free boundary and to limit the domain of $\widetilde{F}$ to a subset of $\mathbb{H}^{1}(S, E)$ composed of mappings whose boundary values are contained in the identity of the homotopy class $\pi_{1}$ of the cylinder $\mathbf{C}$. In fact, such a boundary class can be defined in a generalized sense for $\mathbb{H}^{1}$ (see [17], Chap. 5). However, this class is not closed under the weak topology of $\mathbb{H}^{1}$, and this presents a major difficulty for the proof that a limit of minimizing sequence preserves the boundary condition (see [17], Chap. 5.1, Theorem 4 for a counter-example).

Fortunately, the free boundary in our case is the cylinder and we may use separation of variables to circumvent this problem. Let $U: S \rightarrow E$ be written in the form $U:=$ $\left\{U^{B}, U^{\mathbf{Y}}\right\}$ where $U^{B}: S \rightarrow B$ and $U^{\mathbf{Y}}: S \rightarrow \mathbf{Y}$. Consider the functional (2.6) written as:

$$
\begin{aligned}
\widetilde{F}\left(U^{B}, U^{\mathbf{Y}}\right)= & \frac{1}{2} \int_{S}\left[\gamma \operatorname{Tr}\left(\nabla U^{B}(s)^{T} \nabla U^{B}(s)\right)\right. \\
& \left.+\operatorname{Tr}\left(\nabla U^{\mathbf{Y}}(s)^{T} \nabla U^{\mathbf{Y}}(s)\right)\right] d s+\alpha \mathcal{D}^{2}(\mu, U) .
\end{aligned}
$$

This representation allows us to define the domain of $U^{\mathbf{Y}}$ as $\mathbb{H}^{1}(S, \mathbf{Y})$ without any boundary condition:

$\mathcal{D} O M_{\mathbf{Y}}:=\left\{U^{\mathbf{Y}} \in \mathbb{H}^{1}(S, \mathbf{Y})\right\}$.

The mapping $U^{B}$, on the other hand, must map $S$ onto $B$ such that $\left.U^{B}\right|_{C_{S}}: C_{S} \rightarrow C_{B}:=\partial B$ is a homeomorphism. This corresponds to treating $U_{B}$ as in a Plateau problem with non-free boundary condition. Following the general wisdom for this type of problems, we need the technical 3-point condition (see [17], p. 235) and set:

$$
\begin{aligned}
\mathcal{D} O M_{B}:= & \left\{U^{B} \in \mathbb{H}^{1}(S, B) \cap C^{0}\left(C_{S}, C_{B}\right)\left|U^{B}\right|_{C_{S}}:\right. \\
& C_{S} \rightarrow C_{B} \text { is a homeomorphism } \\
& \text { such that } U^{B}\left(s_{i}\right)=\zeta_{i} \text { for } i=1,2,3 \text {, where } \\
& s_{1}, s_{2}, s_{3} \text { are three distinct points on } C_{S}, \\
& \text { while } \zeta_{1}, \zeta_{2}, \zeta_{3} \text { are three distinct points on } C_{B} \\
& \text { with the same ordering }\}
\end{aligned}
$$

To show the next fact, we use the $\varepsilon$-conformal mapping theorem of Morrey ([34], pp. 814-815) which, in our present notation, reads:

Theorem 2.2 For a mapping $U \in \mathbb{H}^{1}(S, E) \cap C^{0}\left(C_{S}, C\right)$, for every $\varepsilon>0$ there exists a $C^{2}$ homeomorphism $\tau_{\varepsilon}$ from $\bar{S}$ onto itself which satisfies

$$
Z_{\varepsilon}:=U \circ \tau_{\varepsilon} \in \mathbb{H}^{1}(S, E) \cap C^{0}\left(C_{S}, C\right)
$$

and

$\widetilde{A}\left(Z_{\varepsilon}\right) \leq A(U)+\varepsilon$

where $A$ and $\widetilde{A}$ are defined as in (2.2) and (2.4) respectively. 
For proof see [34].

The following theorem is the main goal of this section.

Theorem 2.3 If $\mu$ has a compact support in $E$ then a minimizer of $\widetilde{F}$ is attained in the domain $\mathcal{D} O M:=\mathcal{D} O M_{B} \times$ $\mathcal{D} O M_{\mathbf{Y}}$. Moreover, any minimizer $\bar{U}$ is a minimizer of $F$ (2.3) as well.

For proof see Appendix A.2.

\subsection{Relaxation of the Distance Function}

In this section we define the relaxed distance $D$, denoted by $D_{\beta}$ for a small parameter $\beta>0$. We also show that $D$ is the $\Gamma$-limit of $D_{\beta}$ as $\beta \searrow 0$.

Definition 2.1 Let $(S, d)$ be a separable metric space and let $F_{n}: S \rightarrow[0,+\infty], n=1,2, \ldots$ be a sequence of functions. The sequence $F_{n}$ is said to $\Gamma$-converge to $F: S \rightarrow[0,+\infty]$ if the following two conditions hold for all $f \in S$ :

$$
\begin{aligned}
& \forall f_{n} \rightarrow f \quad \liminf _{n \rightarrow \infty} F_{n}\left(f_{n}\right) \geq F(f), \quad \text { and } \\
& \exists f_{n} \rightarrow f \quad \limsup _{n \rightarrow \infty} F_{n}\left(f_{n}\right) \leq F(f) .
\end{aligned}
$$

First let define a relaxation of the $\sup _{v}$ and $\inf _{\mu}$ functionals (2.9) and (2.10). Given $\beta>0$, set

$$
\begin{aligned}
& \sup _{v}^{\beta}(f)=\beta \ln \left[\int_{E} e^{f(v) / \beta} v(d v)\right], \\
& \underset{\mu}{\beta}(f)=-\beta \ln \left[\int_{E} e^{-f(u) / \beta} \mu(d u)\right] .
\end{aligned}
$$

Lemma 2.2 For a fixed continuous function $f$ in $E$ and fixed Borel measures $\mu$ and $v$ supported in $E$ :

$$
\begin{aligned}
& \lim _{\beta \rightarrow 0} \sup _{v}^{\beta}(f)=\sup _{v}(f), \\
& \liminf _{\beta \rightarrow 0} \inf _{\mu}(f)=\inf _{\mu}(f) .
\end{aligned}
$$

Moreover, if $v_{n}$ converges $C^{*}$ to $v$ and $\beta_{n} \rightarrow 0$ from above $\left(\beta_{n} \geq 0\right)$, then

$$
\liminf _{n \rightarrow \infty} \sup _{v_{n}}^{\beta_{n}}(f) \geq \sup _{v}(f) .
$$

For proof see Appendix A.3.

Let define now

$$
\begin{aligned}
q_{\beta}(v) & :=\inf _{\mu}^{\beta}\left(|u-v|^{2}\right) \\
& =-\beta \ln \left[\int_{E} e^{-|u-v|^{2} / \beta} \mu(d u)\right]
\end{aligned}
$$

and

$\sup _{v}^{\beta}\left(q_{\beta}\right)=\beta \ln \left[\int_{E} e^{q_{\beta}(v) / \beta} v(d v)\right]$.

Now, inserting (2.19) into (2.20) we define the relaxation $D_{\beta}$ of $D$ as:

$D_{\beta}(\mu, v)=\sqrt{\beta \ln \left[\int_{E} \frac{v(d v)}{\int_{E} e^{-|u-v|^{2} / \beta} \mu(d u)}\right]}$.

Using Lemma 2.2 we obtain:

Corollary 2.3 $D(\mu, \cdot)$ is the $\Gamma$-limit of $D_{\beta}(\mu, \cdot)$ as $\beta \rightarrow$ 0 from above, if considered as a functional of the second argument. That is:

$\lim _{\beta \rightarrow 0 ; \beta>0} D_{\beta}(\mu, v)=D(\mu, v)$,

$\liminf _{n \rightarrow \infty} D_{\beta_{n}}\left(\mu, v_{n}\right) \geq D(\mu, \nu)$,

where $\beta_{n} \rightarrow 0$ from above and $v_{n} \rightarrow v$ in $C^{*}$.

Proof Follows immediately from Lemma 2.2 and the fact that $q_{\beta}(v)$ is continuous.

Remark Corollary 2.3 still holds if we assume $\beta \rightarrow 0$ from below. However, in this case, the $\Gamma-$ limit is not to $D$ as defined by (2.8), but to $D_{1}(\mu, v)=d_{1}(\operatorname{supp}(\mu), \operatorname{supp}(v))$ where

$d_{1}(A, B)=\sqrt{\inf _{v \in A} \sup _{u \in B}|u-v|^{2}}$.

In particular,

$\lim _{\beta \rightarrow 0 ; \beta>0} D_{\beta}(\mu, \nu) \neq \lim _{\beta \rightarrow 0 ; \beta<0} D_{\beta}(\mu, \nu)$

in general(!). It is easy to see that $D_{1}$ is also $C^{*}$ lower semicontinuous with respect to $v$. However, it is not a good distance function. Note that $D_{1}(\mu, v)>0$ if $\operatorname{supp}(v)$ is not a single point and $\operatorname{supp}(v) \subset \operatorname{supp}(\mu)$, while $D(\mu, v)=0$ under the above conditions.

\subsection{Relaxation of the Original Functional}

Here we introduce an implementation of the relaxed distance function $D_{\beta}$ and define a corresponding version of the metric $\mathcal{D}_{\beta}(\mu, U), U \in \mathbb{H}^{1}(S, E)$. This enables us to introduce the relaxation of $\widetilde{F}(2.6)$ by

$$
\widetilde{F}_{\beta}(U)=\frac{1}{2} \int_{S} \operatorname{Tr}\left(\nabla U(s)^{T} h \nabla U(s)\right) d s+\alpha \mathcal{D}_{\beta}^{2}(\mu, U) .
$$


We set the $\beta$-distance between a mapping $U: S \rightarrow E$ to a measure $\mu$ as

$\mathcal{D}_{\beta}(\mu, U):=D_{\beta}\left(\mu, \nu_{U}\right)$

$$
=\sqrt{\beta \ln \left[\int_{S} \frac{d s}{\int_{E} e^{-|U(s)-z|^{2} / \beta} \mu(d z)}\right]} .
$$

Thus

$\mathcal{D}_{\beta}^{2}(\mu, U)=\beta \ln \left[\int_{S} \Xi_{\beta}(U)(s) d s\right]$,

where

$\Xi_{\beta}(U)(s)=\frac{1}{\int_{E} e^{-|U(s)-z|^{2} / \beta} \mu(d z)}$.

We now claim:

Corollary $2.4 \mathcal{D}(\mu, \cdot)$ is the $\Gamma$-limit of $\mathcal{D}_{\beta}(\mu, \cdot)$ as $\beta \rightarrow 0$ from above.

Proof Follows immediately from Corollary 2.3 and Proposition 2.1.

We may now write explicitly the functional (2.21):

$$
\begin{aligned}
\widetilde{F}_{\beta}(U)= & \frac{1}{2} \int_{S} \operatorname{Tr}\left(\nabla U(s)^{T} h \nabla U(s)\right) d s \\
& +\alpha \beta \ln \left[\int_{S} \Xi_{\beta}(U)(s) d s\right] .
\end{aligned}
$$

The following theorem is the main goal of this section.

Theorem 2.4 For any $\beta>0$, the functional (2.23) attains a minimizer $U_{\beta} \in \mathcal{D} O M$. The sequence of minimizers $\left\{U_{\beta}\right\}$ is bounded in $\mathbb{H}^{1}(S, E)$. Any weak limit $U$ in $\mathbb{H}^{1}$, s.t.

$U_{\beta} \stackrel{\beta \rightarrow 0}{\rightarrow} U \in \mathcal{D} O M$

is a minimizer of $F(2.3)$ and $\widetilde{F}$ and is a conformal embedding of $S$ into $E$.

For proof see Appendix A.4.

The functional $\widetilde{F}_{\beta}$ equipped with the relaxed distance $\mathcal{D}_{\beta}$ is no longer parameter independent. In particular, its minimizers are not conformal mappings. However, it is converging to parameter independent functional $\widetilde{F}$ as $\beta \rightarrow 0$.

\section{Parametric Representation}

3.1 Variational Formulation and Euler-Lagrange Equation for the Relaxed Functional

Let consider a parametric representation of the image $U$. In this case also $U(S):=\left\{U^{B}, U^{\mathbf{Y}}\right\}$, where $U^{B}$ is the projec- tion of $U$ on the pixel space $B$ and $U^{\mathbf{Y}}$ is the projection of $U$ on the feature fiber $\mathbf{Y}$. We also separate the scale $\gamma$ into $\gamma_{1}$ for the area term and $\gamma_{2}$ for the metric. For such formulation the area term of functional (2.23) is given as in (2.4):

$$
\begin{aligned}
\widetilde{A}(U) & =\frac{1}{2} \int_{S} \operatorname{Tr}\left(\nabla U(s)^{T} h \nabla U(s)\right) d s \\
& =\frac{1}{2} \int_{S}\left(\left\|U_{s_{1}}\right\|_{\gamma_{1}}^{2}+\left\|U_{s_{2}}\right\|_{\gamma_{1}}^{2}\right) d s,
\end{aligned}
$$

where

$$
\begin{aligned}
\|V\|_{\gamma} & =\sqrt{\gamma\left(V^{1}\right)^{2}+\gamma\left(V^{2}\right)^{2}+\sum_{k=3}^{m+2}\left(V^{k}\right)^{2}} \\
& =\sqrt{\gamma\left(V^{B}\right)^{2}+\left(V^{\mathbf{Y}}\right)^{2}} .
\end{aligned}
$$

Let now define the relaxed metric term $\mathcal{D}_{\beta, \gamma_{2}}(\mu, U)$. The parameter dependent metric (2.22) attributes the measure $d s$ to the image $U$, and it is written as

$\mathcal{D}_{\beta, \gamma_{2}}^{2}(\mu, U)=\beta \ln \left[\int_{S} \Xi_{\beta, \gamma_{2}}(U)(s) d s\right]$,

where

$\Xi_{\beta, \gamma_{2}}(U)(s)=\frac{1}{\int_{E} e^{-\|U(s)-z\|_{\gamma_{2}}^{2} / \beta} \mu(d z)}$.

For a parametric formulation we represent the data $\mu(d z)$ as $\mu_{b}(d y) d b$. If, in addition, we restrict ourselves to a deterministic data $G \in \mathbb{L}^{\infty}(B, \mathbf{Y})$, meaning $\mu_{b}(d y):=\delta(y-$ $G(b))$, and utilize the decomposition $U=\left(U^{B}, U^{\mathbf{Y}}\right)$ of (2.13), we obtain

$$
\begin{aligned}
\Xi_{\beta, \gamma_{2}}(U)(s)= & {\left[\int _ { B } \operatorname { e x p } \left(-\beta^{-1}\left(\gamma_{2}\left|U^{B}(s)-b\right|^{2}\right.\right.\right.} \\
& \left.\left.\left.+\left|U^{\mathbf{Y}}(s)-G(b)\right|^{2}\right)\right) d b\right]^{-1} .
\end{aligned}
$$

Finally, the corresponding functional is:

$$
\begin{aligned}
\mathbf{F}_{\gamma_{1}, \gamma_{2}}^{\beta}(U)= & \frac{1}{2} \int_{S}\left(\left\|U_{s_{1}}\right\|_{\gamma_{1}}^{2}+\left\|U_{s_{2}}\right\|_{\gamma_{1}}^{2}\right) d s \\
& +\alpha \beta \ln \left[\int_{S} \Xi_{\beta, \gamma_{2}}(U)(s) d s\right],
\end{aligned}
$$

where $\Xi_{\beta, \gamma_{2}}(U)(s)$ is given as in (3.3).

The Euler-Lagrange equations associated with the functional $\mathbf{F}_{\gamma_{1}, \gamma_{2}}^{\beta}$ can be calculated directly from

$\mathcal{D} \mathbf{F}_{\gamma_{1}, \gamma_{2}}^{\beta}(U) \circ W:=\left.\frac{d}{d \eta} \mathbf{F}_{\gamma_{1}, \gamma_{2}}^{\beta}(U+\eta W)\right|_{\eta=0} \equiv 0$, 
for any admissible perturbation $W$. Using the decomposition $U=\left(U^{B}, U^{\mathbf{Y}}\right)$ as in (2.13) we readily calculate $\mathcal{D} \mathbf{F}_{\gamma_{1}, \gamma_{2}}^{\beta}(U)$ to obtain

$$
\left\{\begin{array}{l}
\Delta U^{B}=\frac{\gamma_{2}}{\gamma_{1}} \cdot \frac{2 \alpha}{\int_{S} \Xi_{\beta, \gamma_{2}}(U)(s) d s} f^{B}(U) \\
\Delta U^{\mathbf{Y}}=\frac{2 \alpha}{\int_{S} \Xi_{\beta, \gamma_{2}}(U)(s) d s} f^{\mathbf{Y}}(U)
\end{array},\right.
$$

where

$$
f(U):=\left\{\begin{aligned}
f^{B}(U):= & \left(\Xi_{\beta, \gamma_{2}}(U)(s)\right)^{2} \\
& \times \int_{B} e^{-\beta^{-1}\left[\gamma_{2}\left|U^{B}(s)-b\right|^{2}+\left|U^{\mathbf{Y}}(s)-G(b)\right|^{2}\right]} \\
& \times\left(U^{B}(s)-b\right) d b, \\
f^{\mathbf{Y}}(U):= & \left(\Xi_{\beta, \gamma_{2}}(U)(s)\right)^{2} \\
& \times \int_{B} e^{-\beta^{-1}\left[\gamma_{2}\left|U^{B}(s)-b\right|^{2}+\left|U^{\mathbf{Y}}(s)-G(b)\right|^{2}\right]} \\
& \times\left(U^{\mathbf{Y}}(s)-G(b)\right) d b .
\end{aligned}\right.
$$

The natural boundary conditions are different for both components of $U$. While $U^{\mathbf{Y}}$ admits no further constraints via (2.14), it satisfies the homogeneous Neumann conditions

$\left.\frac{\partial U^{\mathbf{Y}}}{\partial \vec{v}}\right|_{\partial S}=0 \quad$ on $\partial S$

where $v$ is the normal to $\partial S$. The boundary condition associated with $U^{B}$, on the other hand, is a nonlinear one since (2.15) implies that

$U_{B}: \partial S \rightarrow \partial B$

\subsection{Interior Regularity of the Minimizer of Relaxed \\ Functional}

In Sect. 2.5 we have proved that for any $\beta>0$, the functional (3.4) attains a minimizer $U_{\beta} \in \mathcal{D} O M$, i.e. the system (3.6) has a weak solution $U_{\beta} \in \mathbb{H}^{1}(S, E)$. We now address the question of regularity of such a minimizer. In fact, in this section we intend to show the infinite interior differentiability of the minimizer $U_{\beta}$.

Theorem 3.1 If $U_{\beta}$ is a weak solution of (3.5) subjected to the boundary conditions (3.7)-(3.8) and, in addition, is a minimizer of (3.4) on DOM, then $U_{\beta} \in C^{\infty}(S, E)$.

Note that it does not imply boundary regularity. We prove Theorem 3.1 below.

Proposition 3.1 Let $U_{\beta} \in \mathbb{H}^{1}(S, E)$ be a minimizer of the functional (3.4), then $U_{\beta} \in \mathbb{L}^{\infty}(S, E)$, if $G \in \mathbb{L}^{\infty}(B, \mathbf{Y})$. Moreover, $\left\|U_{\beta}^{\mathbf{Y}}\right\|_{\infty} \leq\|G\|_{\infty}$.
Proof It is obvious that $U_{\beta}^{B} \in \mathbb{L}^{\infty}(S, B)$. Suppose by contradiction that $U_{\beta}^{\mathbf{Y}} \notin \mathbb{L}^{\infty}(S, \mathbf{Y})$. Denote $R=\|G\|_{\infty}$ and define the retraction

$\tilde{U}_{\beta}^{\mathbf{Y}}(s):=\left\{\begin{array}{l}R \frac{U_{\beta}^{\mathbf{Y}}(s)}{\left|U_{\beta}^{\mathbf{Y}}(s)\right|},\left|U_{\beta}^{\mathbf{Y}}(s)\right| \geq R, \\ U_{\beta}^{\mathbf{Y}}(s),\left|U_{\beta}^{\mathbf{Y}}(s)\right|<R\end{array} \quad \tilde{U}_{\beta}^{B} \equiv U_{\beta}^{B}\right.$.

From the definition of retraction it is obvious that

$\left\|\frac{\partial \tilde{U}_{\beta}^{\mathbf{Y}}}{\partial s_{1}}\right\|_{\gamma_{1}}^{2}+\left\|\frac{\partial \tilde{U}_{\beta}^{\mathbf{Y}}}{\partial s_{2}}\right\|_{\gamma_{1}}^{2}<\left\|\frac{\partial U_{\beta}^{\mathbf{Y}}}{\partial s_{1}}\right\|_{\gamma_{1}}^{2}+\left\|\frac{\partial U_{\beta}^{\mathbf{Y}}}{\partial s_{2}}\right\|_{\gamma_{1}}^{2}$

and

$\left|\tilde{U}^{\mathbf{Y}}(s)-G(b)\right| \leq\left|U^{\mathbf{Y}}(s)-G(b)\right|$,

thus,

$\Xi_{\beta, \gamma_{2}}\left(\tilde{U}_{\beta}\right)(s)<\Xi_{\beta, \gamma_{2}}\left(U_{\beta}\right)(s)$.

Taking together (3.9) and (3.10), we obtain that

$\mathbf{F}_{\gamma_{1}, \gamma_{2}}^{\beta}\left(\tilde{U}_{\beta}\right)<\mathbf{F}_{\gamma_{1}, \gamma_{2}}^{\beta}\left(U_{\beta}\right)$,

which contradicts the assumption that $U_{\beta}$ is the minimizer of $\mathbf{F}_{\gamma_{1}, \gamma_{2}}^{\beta}(U)$.

We now prove upper estimates for $\Xi_{\beta, \gamma_{2}}\left(U_{\beta}\right)$ and $\left(\int_{S} \Xi_{\beta, \gamma_{2}}\left(U_{\beta}\right)(s) d s\right)^{-1}$. Assume, as in the Proposition 3.1, that $\|G\|_{\infty}=R$ and consequently, $\left\|U_{\beta}^{\mathbf{Y}}\right\|_{\infty} \leq R$. Denoting $r=\frac{1}{2} \operatorname{diam}(B)$ and using

$\gamma_{2}\left|U_{\beta}^{B}(s)-b\right|^{2}+\left|U_{\beta}^{\mathbf{Y}}(s)-G(b)\right|^{2} \leq 4\left(\gamma_{2} r^{2}+R^{2}\right)$,

we have

$$
\begin{aligned}
\Xi_{\beta, \gamma_{2}}\left(U_{\beta}\right)(s) & \leq \frac{1}{\int_{B} e^{-4\left(\gamma_{2} r^{2}+R^{2}\right) / \beta} d b} \\
& =\frac{1}{|B|} e^{4\left(\gamma_{2} r^{2}+R^{2}\right) / \beta},
\end{aligned}
$$

in particular $\Xi_{\beta, \gamma_{2}}\left(U_{\beta}\right) \in \mathbb{L}^{\infty}(S)$. Taking into account the following inequality

$$
\begin{aligned}
& \gamma_{2}\left|U_{\beta}^{B}(s)-b\right|^{2}+\left|U_{\beta}^{\mathbf{Y}}(s)-G(b)\right|^{2} \\
& \geq(1-\varepsilon)\left(\gamma_{2}\left|U_{\beta}^{B}(s)\right|^{2}+\left|U_{\beta}^{\mathbf{Y}}(s)\right|^{2}\right) \\
&+\left(1-\varepsilon^{-1}\right)\left(\gamma_{2}|b|^{2}+|G(b)|^{2}\right),
\end{aligned}
$$

for $\varepsilon=\frac{1}{2}$ we obtain 


$$
\begin{aligned}
\Xi_{\beta, \gamma_{2}}\left(U_{\beta}\right)(s) & \geq \frac{e^{\left|U_{\beta}(s)\right|_{\gamma_{2}}^{2} / 2 \beta}}{\int_{B} e^{\left(\gamma_{2}|b|^{2}+|G(b)|^{2}\right) / \beta} d b} \\
& \geq \frac{1}{\int_{B} e^{\left(\gamma_{2} r^{2}+R^{2}\right) / \beta} d b}=\frac{1}{|B| e^{\left(\gamma_{2} r^{2}+R^{2}\right) / \beta}},
\end{aligned}
$$

and thus,

$$
\left(\int_{S} \Xi_{\beta, \gamma_{2}}\left(U_{\beta}\right)(s) d s\right)^{-1} \leq \frac{|B|}{\pi} e^{\left(\gamma_{2} r^{2}+R^{2}\right) / \beta} .
$$

(3.5) and (3.6) now imply:

Corollary 3.1 Let $U_{\beta} \in \mathbb{L}^{\infty}(S, E)$. Then $f\left(U_{\beta}\right) \in \mathbb{L}^{\infty}(S)$.

To show the next fact, we use the interior $\mathbb{H}^{2}$-regularity theorem (see [18], Sect. 6.3.1, Theorem 1), which, in our present notation, reads:

Theorem 3.2 Assume that $u \in \mathbb{H}^{1}(S)$ is a weak solution of the elliptic PDE

$\Delta u=f \quad$ in $S$,

and $f \in \mathbb{L}^{2}(S)$. Then $u \in \mathbb{H}_{\text {loc }}^{2}(S)$.

For proof see [18].

It follows that there exists the weak solution $U_{\beta} \in$ $\mathbb{H}^{1}(S, E)$ of (3.5), s.t.

$U_{\beta} \in \mathbb{H}_{\text {loc }}^{2}(S, E)$.

We now use the higher interior regularity theorem ([18], Sect. 6.3.1, Theorem 2) and general Sobolev inequalities theorem ([18], Sect. 5.6.3, Theorem 6), which, in our present notation, read respectively in a following manner:

Theorem 3.3 Assume that $u \in \mathbb{H}^{1}(S, E)$ is a weak solution of the elliptic PDE

$\Delta u=f \quad$ in $S$,

and $f \in \mathbb{H}^{n}(S)$. Then $u \in \mathbb{H}_{\text {loc }}^{n+2}(S)$.

For proof see [18].

Theorem 3.4 Let $S$ be a bounded open subset of $\mathbb{R}^{2}$, with $C^{1}$ boundary. Assume $u \in \mathbb{H}_{\text {loc }}^{n}(S), n \geq 2$. Then $u \in$ $C^{n-2}(S)$.

For proof see [18].

The following proposition includes an induction assumption and its proof.
Proposition 3.2 Assume that there exists a weak solution $U_{\beta}$ of $(3.5)$, s.t. $U_{\beta} \in \mathbb{H}_{l o c}^{n}(S, E) \cap C^{n-1}(S, E)$. Then $U_{\beta}, \Xi_{\beta, \gamma_{2}}\left(U_{\beta}\right)$ and $e^{-\beta^{-1}\left[\gamma_{2}\left|U_{\beta}^{B}(\cdot)-b\right|^{2}+\mid U_{\beta}^{\mathbf{Y}}(\cdot)-G(b)\right]^{2}}$ for all $b \in B$ are in $\mathbb{H}_{\text {loc }}^{n+1}(S, E) \cap C^{n}(S, E)$.

For proof see [28].

Proof of Theorem 3.1 We now repeatedly apply the Proposition 3.2 for $n=2,3, \ldots$ to deduce the infinite differentiability of $U_{\beta}$ in the interior.

\subsection{Some Properties of the Minimizer of Original}

Functional

In this section we shall discuss some local properties of the minimizer $\bar{U}$ of original functional (2.13):

$\widetilde{F}(U)=\frac{1}{2} \int_{S} \operatorname{Tr}\left(\nabla U(s)^{T} h \nabla U(s)\right) d s+\alpha \mathcal{D}^{2}(\mu, U)$.

Actually, we intend to show here, that $\bar{U}$ is locally Lipschitz continuous in $S$. We shall base our reasoning on Characterization of $W^{1, \infty}$ Theorem ([18], Sect. 5.8.2(b), Theorem 4), that, in our present notation, read as follows:

Theorem 3.5 Let $S$ be open and bounded, with $\partial S$ of class $C^{1}$. Then $U: S \rightarrow E$ is Lipschitz continuous if and only if $U \in W^{1, \infty}(S, E)$.

For proof see [18].

Let $\bar{U}$ be a minimizer of the functional $\widetilde{F}(U)$. By the compactness of $\operatorname{supp}(\mu)$ and the definition of $\mathcal{D}(\mu, U)$ we obtain that $\bar{U}$ is bounded in $\mathbb{L}^{\infty}(S, E)$. We tend to show now that $\nabla \bar{U}$ is also bounded in $\mathbb{L}^{\infty}(S, E)$. To conclude this, we go back to Sect. 2.5 and remind the definition of the sequence of functionals $\mathbf{F}_{\beta, \gamma_{2}}$ converging in $\Gamma$-sense to the original functionals $\widetilde{F}(U)$. By Theorem 2.4 , the sequence of its minimizers $U_{\beta}$ weakly converges in $\mathbb{H}^{1}(S, E)$ to the minimizer of original functional $\bar{U}$. In particular, $\nabla U_{\beta}$ converges weakly $\mathbb{L}^{2}(S, E)$ to $\nabla \bar{U}$.

Next, we utilize the gradient estimates for Poisson's equation ([19], Sect. 3.4, Theorem 3.9):

Theorem 3.6 Let $u \in C^{2}(\Omega)$ satisfy Poisson's equation, $\Delta u=f$, in $\Omega$. Then

$\sup _{\Omega} d_{x}|D u(x)| \leq C\left(\sup _{\Omega}|u|+\sup _{\Omega} d_{x}^{2}|f(x)|\right)$,

where $d_{x}=\operatorname{dist}(x, \partial \Omega)$.

For proof see [19].

The following theorem is the main goal of this section. 
Theorem 3.7 Let $\bar{U}$ be a minimizer of functional (2.13) in the domain $\mathcal{D} O M$. Then $\bar{U}$ is locally Lipschitz continuous in $S$.

For proof see Appendix A.5.

\section{Non-parametric Representation}

4.1 Variational Formulation and Euler-Lagrange Equation for the Relaxed Functional

Let consider a non-parametric representation of the image $U$. In this representation we set $S \equiv B$ and $U(S):=$ $\left\{U_{B}, U_{\mathbf{Y}}\right\}$ given by $U_{B}: B \rightarrow B$ is the identity. Thus, the image is given in terms of a graph $U_{\mathbf{Y}}:=f: B \rightarrow \mathbf{Y}$.

Considering the image $U$ in terms of a graph of function $f$ does not allow existence of edges in form of the surface folding. Moreover, in case of non-parametric formulation we cannot use the majorant area functional $\widetilde{A}(U)$ as defined in (2.4). For such formulation the area functional is given as in $(2.2)$ :

$A(U)=\int_{S}|\Gamma(U)(s)| d s=\int_{B} \sqrt{\gamma^{2}+\gamma|\nabla f|^{2}} d b$.

We also separate the scale $\gamma$ into $\gamma_{1}$ for the area term and $\gamma_{2}$ for the metric. In order to unify the limits $\gamma_{1} \rightarrow 0$ and $\gamma_{1} \rightarrow \infty$ we normalize the area term by $\frac{1+\sqrt{\gamma_{1}}}{\sqrt{\gamma_{1}}}$. Thus, the corresponding functional is

$$
\begin{aligned}
\mathbf{F}_{\gamma_{1}, \gamma_{2}}^{\beta}(f)= & \left(1+\sqrt{\gamma_{1}}\right) \int_{B} \sqrt{\gamma_{1}+|\nabla f|^{2}} d b \\
& +\alpha \mathcal{D}_{\beta, \gamma_{2}}^{2}(\mu, f) .
\end{aligned}
$$

We define now the relaxed metric term $\mathcal{D}_{\beta, \gamma_{2}}(\mu, f)$. For non-parametric formulation we represent the data $\mu(d z)$ as $\mu_{b}(d y) d b$. Under the above assumption, the parameter dependent metric (2.22) attributes the measure $d b$ to the image $f$, and it is written as

$\mathcal{D}_{\beta, \gamma_{2}}^{2}(\mu, f)=\beta \ln \left[\int_{B} \Psi_{\beta, \gamma_{2}}^{f}(b) d b\right]$,

where

$\Psi_{\beta, \gamma_{2}}^{f}(b)=\frac{1}{\int_{\mathbf{Y}} \int_{B} e^{-\left[|f(b)-y|^{2}+\gamma_{2}\left|b-b^{\prime}\right|^{2}\right] / \beta} d b^{\prime} \mu_{b^{\prime}}(d y)}$.

In addition, the following analysis is performed for deterministic data, thus $\Psi_{\beta, \gamma_{2}}^{f}(b)$ in (4.2) is given as

$\Psi_{\beta, \gamma_{2}}^{f}(b)=\frac{1}{\int_{B} e^{-\left[\left|f(b)-g\left(b^{\prime}\right)\right|^{2}+\gamma_{2}\left|b-b^{\prime}\right|^{2}\right] / \beta} d b^{\prime}}$.
Finally, the corresponding functional is:

$$
\begin{aligned}
\mathbf{F}_{\gamma_{1}, \gamma_{2}}^{\beta}(f)= & \left(1+\sqrt{\gamma_{1}}\right) \int_{B} \sqrt{\gamma_{1}+|\nabla f|^{2}} d b \\
& +\alpha \beta \ln \left[\int_{B} \Psi_{\beta, \gamma_{2}}^{f}(b) d b\right],
\end{aligned}
$$

where $\Psi_{\beta, \gamma_{2}}^{f}(b)$ is given as in (4.3).

The Euler-Lagrange equation associated with the functional (4.4) is given by

$$
\begin{aligned}
& -\operatorname{div}\left(\frac{\left(1+\sqrt{\gamma_{1}}\right) \nabla f}{\sqrt{\gamma_{1}+|\nabla f|^{2}}}\right)+\frac{2 \alpha \Psi_{\beta, \gamma_{2}}^{f}(b)}{\int_{B} \Psi_{\beta, \gamma_{2}}^{f}(b) d b} f \\
& \quad-\frac{2 \alpha\left(\Psi_{\beta, \gamma_{2}}^{f}(b)\right)^{2}}{\int_{B} \Psi_{\beta, \gamma_{2}}^{f}(b) d b} \\
& \quad \times \int_{B} g\left(b^{\prime}\right) e^{-\left[\left|f(b)-g\left(b^{\prime}\right)\right|^{2}+\gamma_{2}\left|b-b^{\prime}\right|^{2}\right] / \beta} d b^{\prime} \\
& =0
\end{aligned}
$$

subjected to the Neumann boundary condition

$\frac{\partial f}{\partial \vec{n}}=0 \quad$ on $\partial B$.

Note that the above non-parametric case (4.5) is similar to anisotropic diffusion approach (see, for example, [38]). The function $G(\nabla f)=\frac{\left(1+\sqrt{\gamma_{1}}\right)}{\sqrt{\gamma_{1}+|\nabla f|^{2}}}$ in the first term of the left-hand part of (4.5) may be considered as edge indicator (penalty) function.

\subsection{Segmentation of Grey-Level Images: Choice of} Parameters, Limiting Cases and Numerical Results

The numerical implementation of two-dimensional problem is fully described in Appendix B. In this section we are going to point out the influence of parameters $\beta, \alpha, \gamma_{1}, \gamma_{2}$ on the segmentations results. To demonstrate the performance of the proposed method, two natural images were used (see Fig. 1) in our numerical experiments. We remind that $\beta$ is the convergence parameter and the minimizer of the original (the limit) functional is achieved when $\beta \rightarrow 0$. We also note that the limit $\beta \rightarrow 0$ of (4.4) yields

$$
\begin{aligned}
\mathbf{F}_{\gamma_{1}, \gamma_{2}}^{0}(f)= & \left(1+\sqrt{\gamma_{1}}\right) \int_{B} \sqrt{\gamma_{1}+|\nabla f|^{2}} d b \\
& +\alpha \sup _{b \in B} \inf _{b^{\prime} \in B}\left[\left|f(b)-g\left(b^{\prime}\right)\right|^{2}+\gamma_{2}\left|b-b^{\prime}\right|^{2}\right],
\end{aligned}
$$

which is the original functional for deterministic images. Thus, for our calculations, $\beta$ is taken sufficiently small. We show the role of parameters $\alpha$ and $\gamma_{1}$ on the examples of 
Fig. 1 Real-life test images of a leopard and a zebra
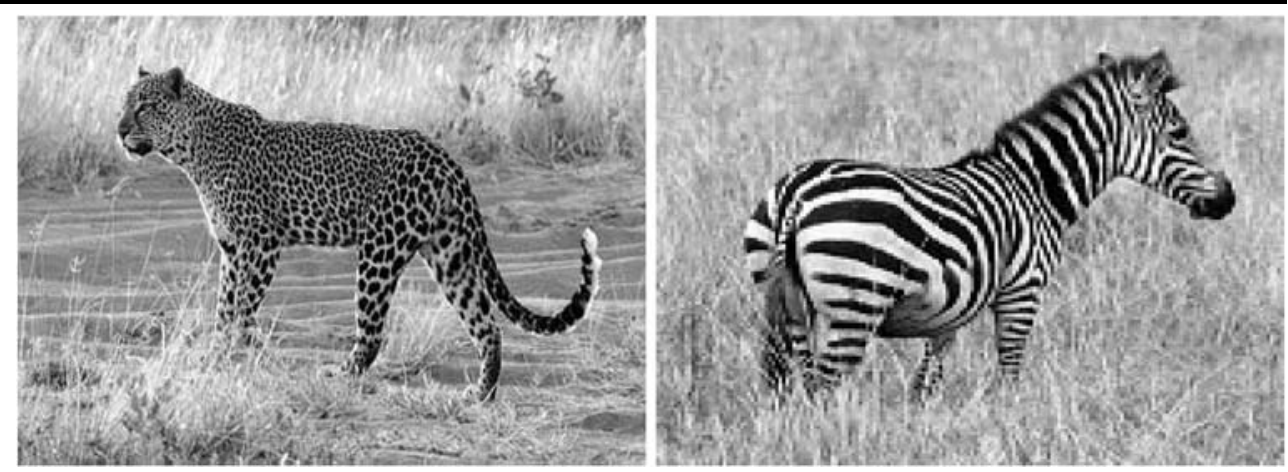

leopard and zebra images respectively, tested in some previous works, especially in [43]. The parameter $\alpha$, as in case of Mumford-Shah functional, measures the trade off between a good fit of the solution $f$ to data $g$ and the regularity of the solution $f$. The result obtained for the leopard image (Fig. 2) in case $\alpha=185$ is comparable to that obtained by Sagiv, Sochen and Zeevi (see [43]).

We define now two limiting cases for the functional (4.4): $\mathbf{F}_{0, \gamma_{2}}^{\beta}(f)$ and $\mathbf{F}_{\infty, \gamma_{2}}^{\beta}(f)$, when $\gamma_{1} \rightarrow 0$ and $\gamma_{1} \rightarrow \infty$, respectively. As $\gamma_{1}$ tends to 0 , one can obtain

$\mathbf{F}_{0, \gamma_{2}}^{\beta}(f)=T V(f)+\alpha \beta \ln \left[\int_{B} \Psi_{\beta, \gamma_{2}}^{f}(b) d b\right]$,

where

$T V(f) \equiv \int_{B}|\nabla f| d b$

is the Total Variation (TV) norm, originally introduced by Rudin et al. in [41]. On the other hand, $\gamma_{1} \rightarrow \infty$ yields

$\mathbf{F}_{\infty, \gamma_{2}}^{\beta}(f) \approx H^{1}(f)+\alpha \beta \ln \left[\int_{B} \Psi_{\beta, \gamma_{2}}^{f}(b) d b\right]$

up to the constant, where

$H^{1}(f) \equiv \int_{B}|\nabla f|^{2} d b$

is Sobolev space norm (see [49]). We remark that the $T V$ norm allows discontinuities in $f$, thus making it superior to the $H^{1}$ regularization in cases where $f$ can have sharp edges. As is shown above, the parameter $\gamma_{1}$ being defined as representing the scale difference between pixel domain and feature fiber, also determines the kind of regularization for function $f$, defining the various regularization norms. The results for various cases of parameter $\gamma_{1}$ may be seen in Fig. 3.

The role of parameter $\gamma_{2}$, which similarly to parameter $\gamma_{1}$ being defined as representing the scale difference between pixel domain and feature fiber, is completely different. Here the case of interest is the limit $\gamma_{2} \rightarrow \infty$. It is easily verified that, as $\gamma_{2} \rightarrow \infty$,

$\lim _{\gamma_{2} \rightarrow \infty} \Psi_{\beta, \gamma_{2}}^{f}(b)=C \frac{\gamma_{2}}{\beta} \cdot e^{|f(b)-g(b)|^{2} / \beta}$,

where $C$ stands for a generic constant. Thus, the limit $\gamma_{2} \rightarrow$ $\infty$ yields

$$
\begin{aligned}
\mathbf{F}_{\gamma_{1}, \infty}^{\beta}(f)= & \lim _{\gamma_{2} \rightarrow \infty} \mathbf{F}_{\gamma_{1}, \gamma_{2}}^{\beta}(f) \\
\approx & \left(1+\sqrt{\gamma_{1}}\right) \int_{B} \sqrt{\gamma_{1}+|\nabla f|^{2}} d b \\
& +\alpha \beta \ln \left[\int_{B} e^{|f(b)-g(b)|^{2} / \beta} d b\right]-\alpha \beta \ln \beta
\end{aligned}
$$

up to the constant multiplied by $\beta$. Finally, we note that the limit $\beta \rightarrow 0$ of (4.7) yields

$$
\begin{aligned}
\mathbf{F}_{\gamma_{1}, \infty}^{0}(f)= & \lim _{\beta \rightarrow 0} \mathbf{F}_{\gamma_{1}, \infty}^{\beta}(f)=\left(1+\sqrt{\gamma_{1}}\right) \int_{B} \sqrt{\gamma_{1}+|\nabla f|^{2}} d b \\
& +\alpha \sup _{b \in B}|f(b)-g(b)|^{2} .
\end{aligned}
$$

It follows that the parameter $\gamma_{2}$ determines the local neighborhood of the pixel $b$, which gives the valuable contribution to the final segmentation image. The smaller $\gamma_{2}$ is, the larger neighborhood of the given pixel influences on its grey level in the output image.

Finally, we present the ideal segmentation for the full zebra image (see Fig. 4), comparable to that obtained by Sagiv, Sochen and Zeevi (see [43]). It is the best result we got for this image. We do not know, at this stage, if the choice of the parameters $\alpha, \gamma_{1}, \gamma_{2}$ and $\beta$ is optimal for this image. However, the effect of the finite parameter $\gamma_{2}$ is demonstrated.

\subsection{Ultrasound Image Segmentation}

Ultrasound imaging is a common, relatively inexpensive and safe modality. It is widely used in medical diagnostics. It allows high acquisition rates and provides images in real-time, but the images are corrupted by the high level of speckle noise. Although the imaging capabilities of modern ultrasound machines are increasing, noise remains a problem 
Fig. 2 Leopard segmentation outputs and outlined original images:

$\beta=0.1, \gamma_{1}=0, \gamma_{2}=\infty$;

$\alpha=185$ in the upper row and

$\alpha=500$ in the lower row

Fig. 3 Zebra segmentation outputs:

$\alpha=330, \beta=0.1, \gamma_{2}=\infty$; $\gamma_{1}=0$ in the upper row on the left, $\gamma_{1}=10$ in the upper row on the right, $\gamma_{1}=\infty$ in the lower row on the left and outlined original image for the last case
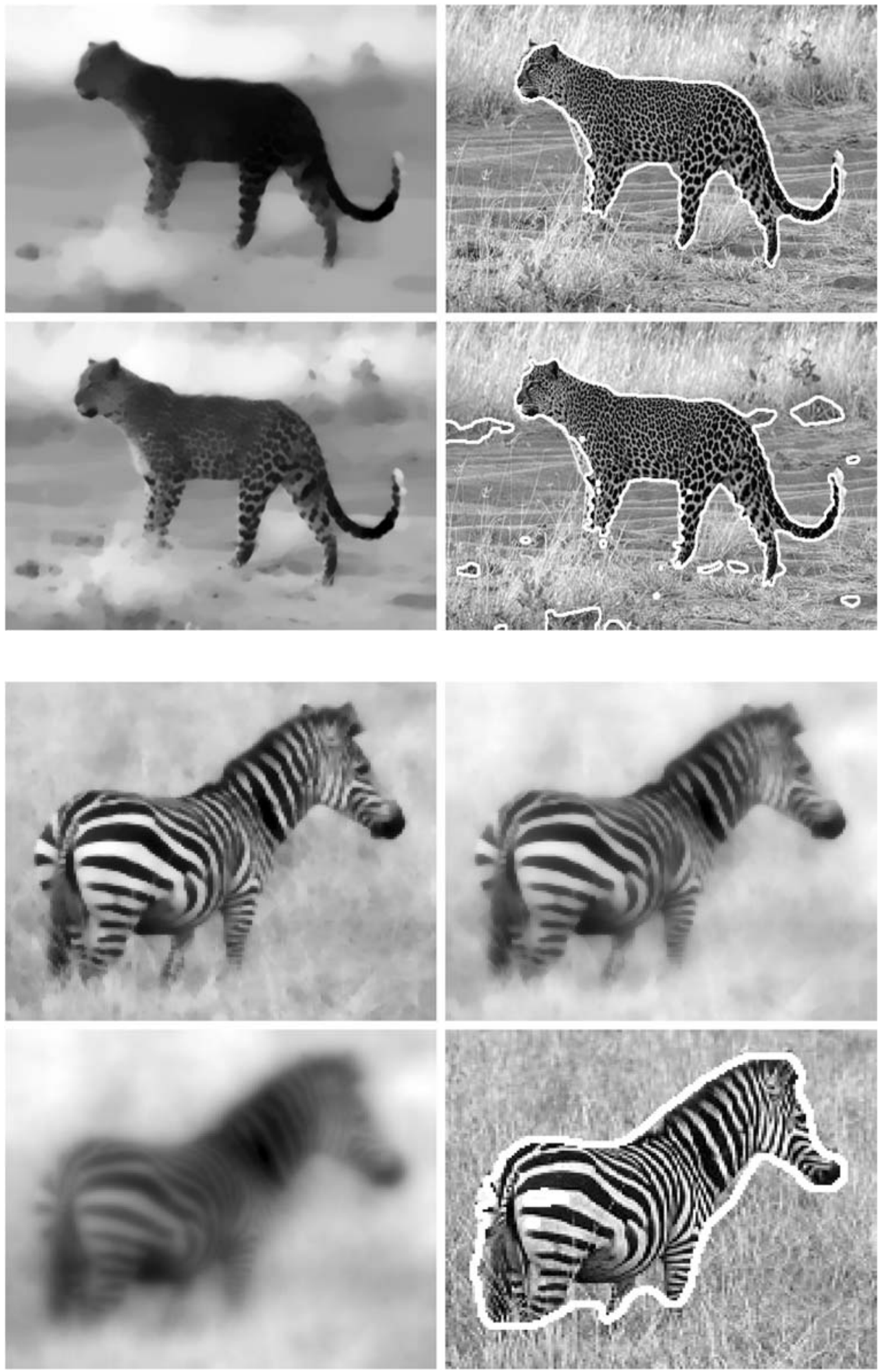

when trying to devise image processing techniques. In fact, noise makes it difficult to accurately identify edges, since in some regions the noise produces artificial edges, while in other regions there are no echoes present and the edges seem ambiguous. In such low-quality images (which are very common in ultrasound imaging), generic algorithms do not identify the border accurately.

Several algorithms have been reported, which could help identify edges in ultrasound images [2, 11, 16, 22]. Studies that are focused on the processing of cardiac ultrasound im- 
Fig. 4 Zebra segmentation output and outlined original image: $\alpha=30000, \beta=10^{-6}$, $\gamma_{1}=0, \gamma_{2}=75000$
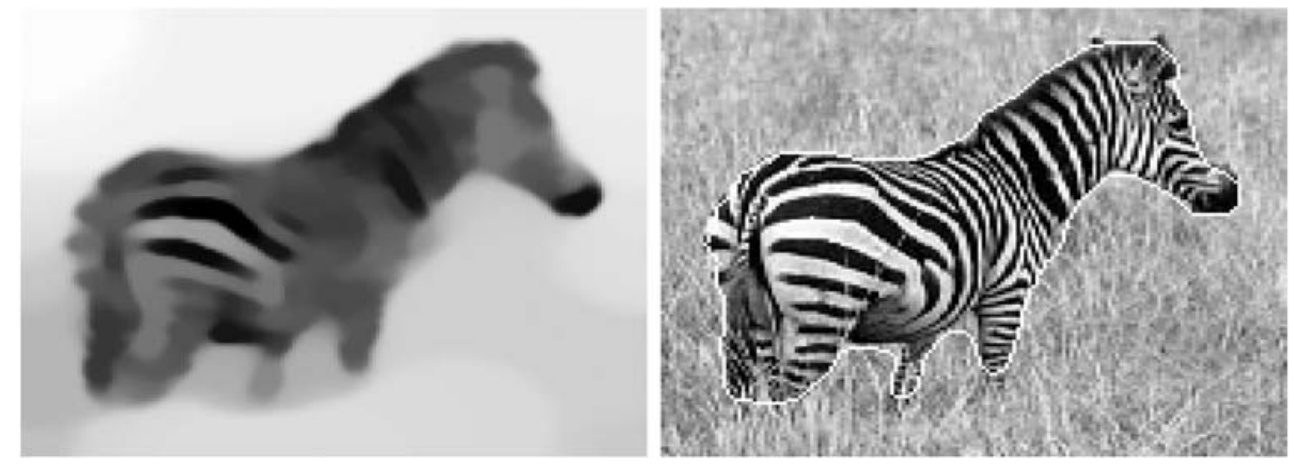

ages $[1,15]$ are also of value, since many of the methods are similar. In addition, a modified version [40] for identifying the lumen-thrombus border in case of aortic aneurysm was developed. Some of these studies are based on active contours [23] or deformable contour [31], some on the snakes algorithm [24], that defines an objective function, also called energy function, which is minimized to obtain the estimated border.

We attempt here to apply our objective function (i.e. relaxed functional) to the ultrasonic images of liver containing some kind of tumor. Ultrasound imaging of the liver is a standard part of a checkup by the specialist in internal medicine. These images are convenient to work with due to their high availability and use in the medical community. The liver is a fairly homogenous organ, in the sense that any given cross-section tends to be fairly uniform. This makes identification of abnormalities somewhat easier. On the other hand, the main difficulty in tumor identification is the fact that the tumor on hepatic ultrasonic images may be presented at different grey levels with respect to other liver tissues. It means that it may be of brighter (see Fig. 5) or darker (see Fig. 7) grey levels then the healthy tissue of liver, or to include wide variety of existing grey levels (see Fig. 6).

The segmentation algorithm is exactly as described in Appendix B, excluding the thresholding of accepted segmentation image by graythresh MATLAB function: Canny edge detector [7] is applied directly to the algorithm output image. Figures 5-7 present the examples of segmented tumor in hepatic ultrasonic images. In all the cases we used TV regularization norm $\left(\gamma_{1}=0\right)$ in order to achieve sharper edges and localized metric term $\left(\gamma_{2}=\infty\right)$ in order to reduce the computation time.

On the Fig. 7 in addition to tumor, one may see also the segmented kidney and gallbladder.

\section{Discussion and Conclusions}

The importance of the functional, proposed in this study, as an alternative to the widely analyzed and implemented functional of Mumford and Shah, is due to the fact that it is independent of parameterization. It is a geometric functional given in terms of the geometry of imbedded surfaces representing the data and image in the feature space. By combining the $\Gamma$-convergence technique with minimal surface theory our approach yielded a global generalization of the Mumford-Shah segmentation functional. Likewise one may consider the implementation of this geometric approach in other image processing and computer vision tasks. In fact, we have illustrated it also in the context of inpainting $[29,30]$.

With reference to segmentation of images, it should be pointed out that there exist a variety of segmentation methods, each being suitable for a specific type of segmentation problem. However, there is no universal algorithm that is optimal for the wide spectrum of images and criteria. The present study is a step towards the development of a general framework that can deal with segmentation problems in the context of multi-channel images.

The main novelty of this study is the replacement of the metric term of Mumford-Shah functional by a completely new type of metric based on Hausdorff distance function. This may be useful in cases of defocusing and of mapping problems, where the relevant errors imply that each pixel point is influenced by a domain rather by a single point of a data. The possible application of the above approach may be image restoration, especially denoising - we implemented it in case of one-dimensional signals, see [28]. Eventually, the proposed change allows us to replace the deterministic data by a random one and include also the case of missing data (inpainting, see [29] and [30]).

Since the new metric term, and thus the functional, suffered from lack of regularity, we utilized an approach adopted from mathematical theory of approximation of functionals via $\Gamma$-convergence to overcome this deficiency. The idea is to approximate the functional by a series of different, parameter-dependent functionals, that are expected to be more regular. However, we should point out that the developed relaxed functional demands extensive computational effort to obtain its minimum. This is the main drawback of our algorithm. An optional solution to this problem is to apply a multi-resolution analysis [6], by performing the 
Fig. 5 Liver tumor

segmentation: $\alpha=650, \beta=0.1$, $\gamma_{1}=0, \gamma_{2}=\infty$; original image in the upper row on the left, segmentation output in the upper row on the right and outlined original image in the lower row
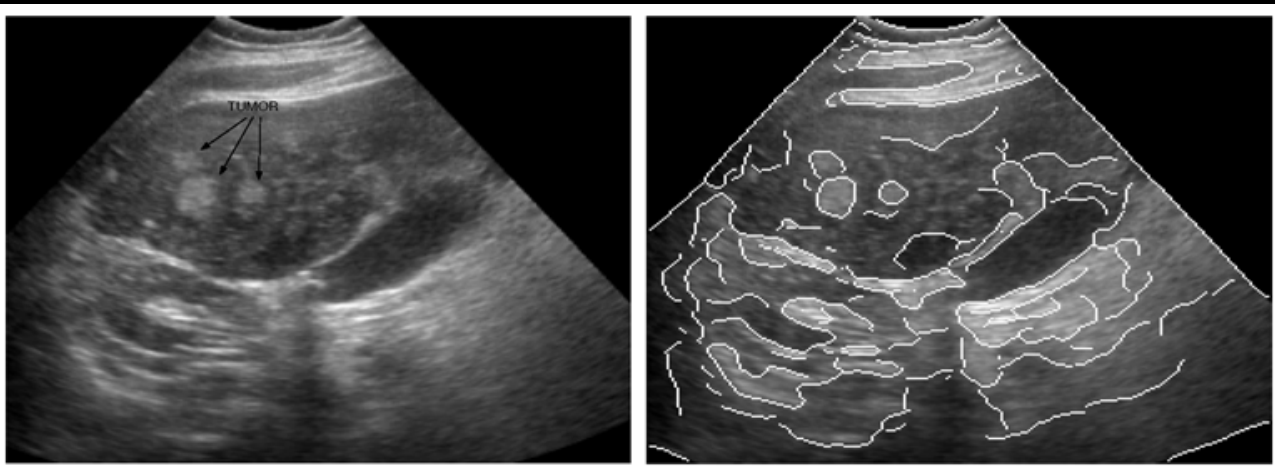

Fig. 6 Liver tumor segmentation: $\alpha=50, \beta=0.1$, $\gamma_{1}=0, \gamma_{2}=\infty$; original image in the upper row on the left, segmentation output in the upper row on the right and outlined original image in the lower row
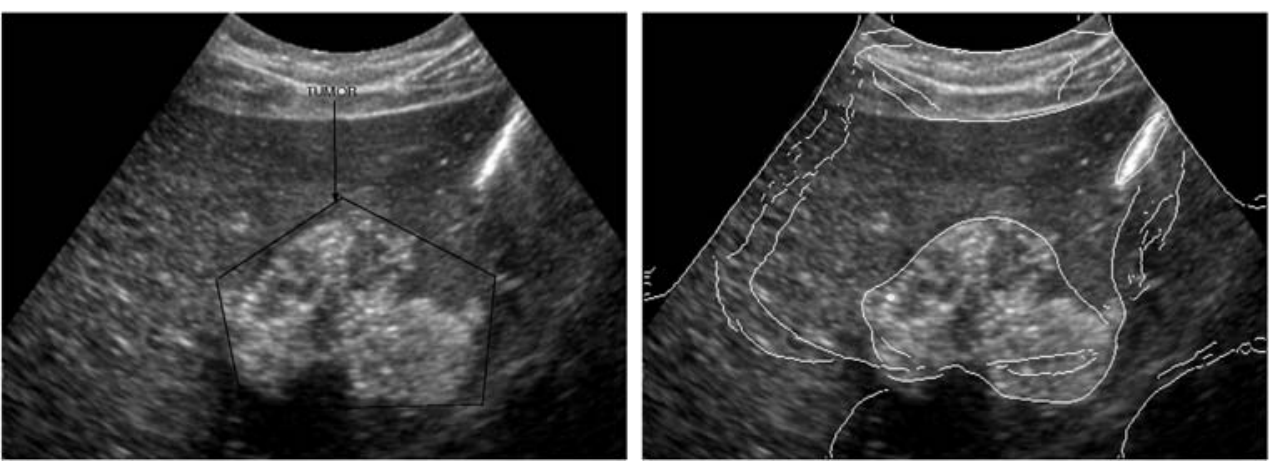

Fig. 7 Liver tumor

segmentation: $\alpha=900, \beta=0.1$, $\gamma_{1}=0, \gamma_{2}=\infty$; original image in the upper row on the left, segmentation output in the upper row on the right and outlined original image in the lower row
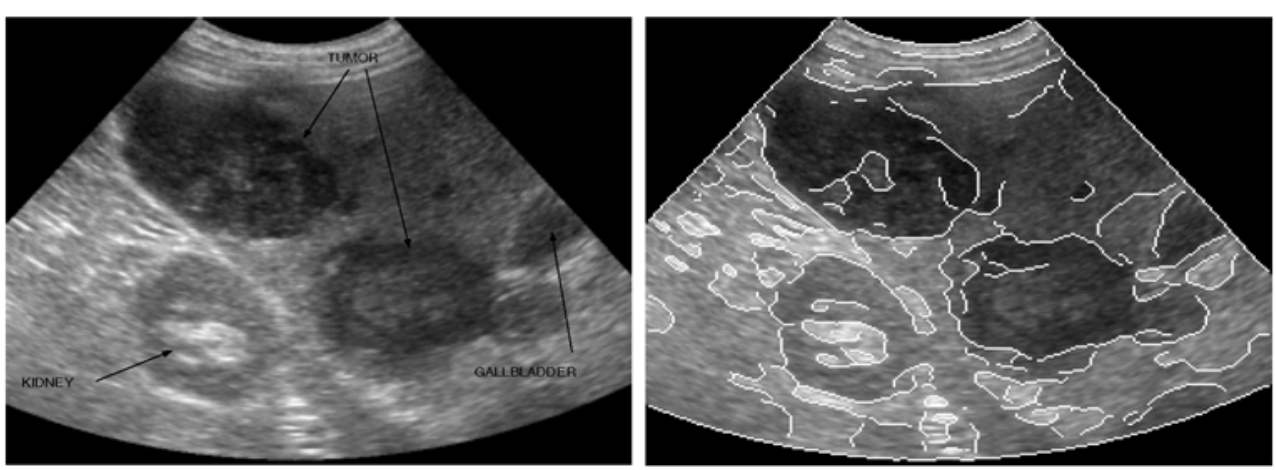

relative computations on higher levels of a Gaussian pyramid and thereby reduce significantly the amount of required computations.

So far we have not touched upon the numerical solution problem of data with parametric representation-the case, whose theoretical developments were introduced in Sect. 4. The parametric representation refers to the case of partially free boundary problem in minimal surface theory. The numerical solution may be obtained by finite element method (see, for example, [48]), where the original variational formulation (and not the PDE as in case of non-parametric representation) is used as a point of departure. This yields, however, a relatively complicated variational formulation (due to the relaxed metric term) of our problem in comparison with one treated in [48]. Obtaining the numerical solution for non-parametric case may be the separate theme for future research.
Acknowledgements This research was supported by the Ollendorff Minerva Center, by the European Union contract HPRN-CT-200300285 (HASSIP), and by the Fund of Promotion of Research at the Technion. We thank Dr. Diana Gaitini, head of the ultrasound unit of the Rambam medical center, for her help and cooperation in the analysis of the ultrasound images.

\section{Appendix A: Proofs of Theorems and Lemmata}

\section{A.1 Proof of Lemma 2.1}

Due to compactness of $C^{*}$ we may isolate a weakly converging subsequence $v_{n}$ to some measure $v$. Denote also by $\bar{v}$ a point in which $f$ attains its supremum within $\operatorname{supp}(v)$ (because $\operatorname{supp}(v)$ is closed). Let us show that for every $\varepsilon>0$ there exists $N \geq 0$, s.t. for $n \geq N \operatorname{supp}\left(v_{n}\right) \cap B_{\varepsilon}(\bar{v}) \neq \emptyset$ $\left(B_{\varepsilon}(\bar{v})\right.$ is an open ball of radius $\varepsilon$ centered at $\left.\bar{v}\right)$. Suppose 
on the contrary, that there exists $\varepsilon>0$ s.t. for every $N$ there exists sequence $n_{k} \geq N$, s.t. $\operatorname{supp}\left(v_{n_{k}}\right) \cap B_{\varepsilon}(\bar{v})=\emptyset$. It follows from here that

$\liminf _{n \rightarrow \infty} v_{n}\left(B_{\varepsilon}(\bar{v})\right)=\lim _{k \rightarrow \infty} v_{n_{k}}\left(B_{\varepsilon}(\bar{v})\right)=0$.

On the other hand, $\operatorname{supp}(v) \cap B_{\varepsilon}(\bar{v}) \neq \emptyset(\bar{v} \in \operatorname{supp}(v))$, thus $\nu\left(B_{\varepsilon}(\bar{v})\right)>0$. Hence,

$\liminf _{n \rightarrow \infty} v_{n}\left(B_{\varepsilon}(\bar{v})\right)<v\left(B_{\varepsilon}(\bar{v})\right)$

and this is a contradiction to the Theorem 2.1: it should be $\liminf _{n \rightarrow \infty} v_{n}\left(B_{\varepsilon}(\bar{v})\right) \geq v\left(B_{\varepsilon}(\bar{v})\right)$.

Recall the original assumption: for every $\varepsilon>0$ there exists $N \geq 0$, s.t. for $n \geq N \operatorname{supp}\left(v_{n}\right) \cap B_{\varepsilon}(\bar{v}) \neq \emptyset$. Hence, there exists the sequence $v_{n} \in \operatorname{supp}\left(v_{n}\right)$, s.t. for $n \geq N$ $\left|v_{n}-\bar{v}\right|<\varepsilon$, i.e. $\lim _{n \rightarrow \infty} v_{n}=\bar{v}$. Taking into account that $\sup _{v \in \operatorname{supp}(v)} f(v) \geq f\left(v_{n}\right)$, we obtain

$$
\begin{aligned}
\liminf _{n \rightarrow \infty} \sup _{v_{n}}(f) & =\liminf _{n \rightarrow \infty} \sup _{v \in \operatorname{supp}\left(v_{n}\right)} f(v) \geq \liminf _{n \rightarrow \infty} f\left(v_{n}\right) \\
& =f(\bar{v})=\sup _{v \in \operatorname{supp}(v)} f(v)=\sup _{v}(f) .
\end{aligned}
$$

\section{A.2 Proof of Theorem 2.3}

Let $U_{n}:=\left\{U_{n}^{B}, U_{n}^{\mathbf{Y}}\right\}$ be a minimizing sequence of $\widetilde{F}$. By the compactness of $\operatorname{supp}(\mu)$ and the definition of $\mathcal{D}$ we obtain that $U_{n}$ are uniformly bounded in $\mathbb{L}^{\infty}(S, E)$. Since the Dirichlet term in $\widetilde{F}$ is bounded as well, we conclude that $U_{n}$ is a bounded sequence in $\mathbb{H}^{1}(S, E)$. Therefore (see [32], Sect. 2.18), there is a weak limit $\bar{U}:=\left\{\bar{U}^{B}, \bar{U}^{\mathbf{Y}}\right\} \in$ $\mathbb{H}^{1}(S, E)$. Thus, we only have to show that $\bar{U}^{B} \in \mathcal{D} O M_{B}$. This follows by the standard proof of the Plateau problem (see, e.g., [17], Chap. 4): We apply the Courant-Lebesgue Lemma and the three-point condition to obtain the equicontinuity of the traces $\left.U_{n}^{B}\right|_{C_{S}}$. The Arzela-Ascoli theorem implies the convergence of the traces to a continuous, weakly monotonic function $\phi: C_{S} \rightarrow C_{B}$ which preserves the three point condition. On the other hand, the traces $\left.U_{n}^{B}\right|_{C_{S}}$ converge in $\mathbb{L}^{2}\left(C_{S}, C_{B}\right)$ by compact embedding of the space of traces $\mathbb{H}^{1 / 2}\left(C_{S}, C_{B}\right)$ in $\mathbb{L}^{2}\left(C_{S}, C_{B}\right)$. This $\mathbb{L}^{2}$ limit of the traces coincides with $\phi$. This implies that $\bar{U}^{B} \in \mathcal{D} O M_{B}$.

Denote $Z_{\varepsilon}=\bar{U} \circ \tau_{\varepsilon}$, where $\tau_{\varepsilon}$ is a $C^{2}$ homeomorphism. Taking an advantage of the fact that $\bar{U}$ is the minimizer of $\widetilde{F}$ and using Theorem 2.2, we obtain

$$
\begin{aligned}
\widetilde{F}(\bar{U}) & \leq \widetilde{F}\left(Z_{\varepsilon}\right)=\widetilde{A}\left(Z_{\varepsilon}\right)+\alpha \mathcal{D}^{2}\left(\mu, Z_{\varepsilon}\right) \\
& =\widetilde{A}\left(Z_{\varepsilon}\right)+\alpha D^{2}\left(\mu, v_{Z_{\varepsilon}}\right)=\widetilde{A}\left(Z_{\varepsilon}\right)+\alpha D^{2}\left(\mu, \nu_{\bar{U}}\right) \\
& \leq A(\bar{U})+\varepsilon+\alpha D^{2}\left(\mu, v_{\bar{U}}\right)=F(\bar{U})+\varepsilon
\end{aligned}
$$

for every $\varepsilon>0$. Passing to the limit $\varepsilon \rightarrow 0$, together with inequality $F(\bar{U}) \leq \widetilde{F}(\bar{U})$ we complete the proof of the theorem.
A.3 Proof of Lemma 2.2

Let show first (2.16). Let $v_{0} \in \operatorname{supp}(v)$ s.t. $\sup _{v} f=f\left(v_{0}\right)$. Then

$\sup _{v}^{\beta}(f)=f\left(v_{0}\right)+\beta \ln \left[\int_{E} e^{\left(f(v)-f\left(v_{0}\right)\right) / \beta} v(d v)\right]$.

Evidently

$\lim _{\beta \rightarrow 0} \beta \ln \left[\int_{E} e^{\left(f(v)-f\left(v_{0}\right)\right) / \beta} v(d v)\right] \leq 0$.

To show the reverse inequality in (A.1), let $B_{\varepsilon}\left(v_{0}\right)$ be a ball of radius $\varepsilon$ centered at $v_{0}$. By continuity of $f$ we have $\inf _{B_{\varepsilon}\left(v_{0}\right)} f \geq f\left(v_{0}\right)-d(\varepsilon)$, where $\lim _{\varepsilon \rightarrow 0} d(\varepsilon)=0$. Moreover, $v\left(B_{\varepsilon}\left(v_{0}\right)\right)>0$ since $v_{0} \in \operatorname{supp}(v)$. Then

$$
\begin{array}{r}
\beta \ln \left[\int_{E} e^{\left(f(v)-f\left(v_{0}\right)\right) / \beta} v(d v)\right] \\
\geq \beta \ln \left[v\left(B_{\varepsilon}\left(v_{0}\right)\right) e^{-d(\varepsilon) / \beta}\right] \\
=-d(\varepsilon)+\beta \ln \left[v\left(B_{\varepsilon}\left(v_{0}\right)\right)\right] .
\end{array}
$$

Taking now the limit $\beta \rightarrow 0$, we obtain that the limit in (A.2) is no smaller than $-d(\varepsilon)$. Since $\varepsilon$ (hence $d(\varepsilon)$ ) can be chosen arbitrary small, we have the equality in (A.1).

The proof of (2.17) is analogous. To prove (2.18), we proceed as in (A.2), where $v\left(B_{\varepsilon}\left(v_{0}\right)\right)$ is replaced by $v_{n}\left(B_{\varepsilon}\left(v_{0}\right)\right)$, and note that $\lim _{n \rightarrow \infty} v_{n}\left(B_{\varepsilon}\left(v_{0}\right)\right)=v\left(B_{\varepsilon}\left(v_{0}\right)\right)$.

\section{A.4 Proof of Theorem 2.4}

Let $U_{n}$ be a minimizing sequence of $\widetilde{F}_{\beta}(U)$ for fixed $\beta>0$. The proof follows along the line of Theorem 2.3, except for the absence of the a-priori $\mathbb{L}^{\infty}$ estimate of $U_{n}$. However, using

$\left|U_{n}(s)-z\right|^{2} \geq(1-\varepsilon)\left|U_{n}(s)\right|^{2}+\left(1-\varepsilon^{-1}\right)|z|^{2}$,

for $\varepsilon>0$ we have

$\Xi_{\beta}\left(U_{n}\right)(s) \geq \frac{e^{(1-\varepsilon)\left|U_{n}(s)\right|^{2} / \beta}}{\int_{E} e^{-\left(1-\varepsilon^{-1}\right)|z|^{2} / \beta} \mu(d z)}$.

By compactness of $\operatorname{supp}(\mu)$ and the choice $\varepsilon=1 / 2$ we obtain an a-priori estimate

$\int_{S} e^{\left|U_{n}(s)\right|^{2} / 2 \beta} \leq \int_{S} \Xi_{\beta}(U)(s) d s \cdot \int_{E} e^{|z|^{2} / \beta} \mu(d z)$,

which is enough (since we actually need only $\mathbb{L}^{2}$ upper estimate for $U_{n}$ ).

The second part of the theorem follows from Corollary 2.4 . 
A.5 Proof of Theorem 3.7

Recall that $U_{\beta}$ is the weak solution of system of semi-linear elliptic equations (3.5). By Proposition 3.1, Corollary 3.1, (3.12) and Theorem 3.6, the sequence $\nabla U_{\beta}$ is in $\mathbb{L}_{l o c}^{\infty}(S, E)$, and moreover, is uniformly bounded there. Thus, by duality of $\mathbb{L}^{\infty}$ to $\mathbb{L}^{1}$, we state that for every $S^{\prime} \subset \subset S$ there exists $M>0$ for which

$$
\begin{aligned}
\left\|\nabla U_{\beta}\right\|_{\mathbb{L}^{\infty}\left(S^{\prime}, E\right)} & =\sup _{\phi \in \mathbb{L}^{1}\left(S^{\prime}, E\right),\|\phi\|_{\mathbb{L}^{1}=1}} \int_{S^{\prime}} \nabla U_{\beta}(s) \phi(s) d s \\
& \leq M .
\end{aligned}
$$

Weak convergence of $\nabla U_{\beta}$ to $\nabla \bar{U}$ implies

$\lim _{\beta \rightarrow 0} \int_{S^{\prime}} \nabla U_{\beta}(s) \phi(s) d s=\int_{S^{\prime}} \nabla \bar{U}(s) \phi(s) d s$.

From (A.3) and (A.4) we obtain that

$$
\int_{S^{\prime}} \nabla \bar{U}(s) \phi(s) d s \leq M
$$

In particular

$$
\begin{aligned}
\|\nabla \bar{U}\|_{\mathbb{L}^{\infty}\left(S^{\prime} ; E\right)} & =\sup _{\phi \in \mathbb{L}^{1}\left(S^{\prime} ; E\right),\|\phi\|_{\mathbb{L}^{1}=1}} \int_{S^{\prime}} \nabla \bar{U}(s) \phi(s) d s \\
& \leq M .
\end{aligned}
$$

We conclude that $\bar{U} \in W_{\text {loc }}^{1, \infty}(S, E)$. Thus, by Theorem 3.5, $\bar{U}$ is locally Lipschitz continuous in $S$.

\section{Appendix B: Numerical Implementation of Two-Dimensional Problem}

Consider now the $M \times N$ image ( $M$ rows and $N$ columns). Let $B \subset \mathbb{R}^{2}$ be its domain and $g(b), b \in B$ denote its graph. We assume that its desired segmentation should be the minimum of relaxed functional (4.4), i.e.

$$
\begin{aligned}
\mathbf{F}_{\gamma_{1}, \gamma_{2}}^{\beta}(f)= & \left(1+\sqrt{\gamma_{1}}\right) \int_{B} \sqrt{\gamma_{1}+|\nabla f|^{2}} d b \\
& +\alpha \beta \ln \left[\int_{B} \Psi_{\beta, \gamma_{2}}^{f}(b) d b\right],
\end{aligned}
$$

where $\Psi_{\beta, \gamma_{2}}^{f}(b)$ is given as in (4.3). We are looking now for a weak solution of the following Euler-Lagrange equation

$$
\begin{aligned}
- & \operatorname{div}\left(\frac{\left(1+\sqrt{\gamma_{1}}\right) \nabla f}{\sqrt{\gamma_{1}+|\nabla f|^{2}}}\right)+\frac{2 \alpha \Psi_{\beta, \gamma_{2}}^{f}(b)}{\int_{B} \Psi_{\beta, \gamma_{2}}^{f}(b) d b} f(b) \\
- & \frac{2 \alpha\left(\Psi_{\beta, \gamma_{2}}^{f}(b)\right)^{2}}{\int_{B} \Psi_{\beta, \gamma_{2}}^{f}(b) d b} \\
& \times \int_{B} g\left(b^{\prime}\right) e^{-\left[\left|f(b)-g\left(b^{\prime}\right)\right|^{2}+\gamma_{2}\left|b-b^{\prime}\right|^{2}\right] / \beta} d b^{\prime}=0
\end{aligned}
$$

with natural boundary conditions

$$
\left.\frac{\partial f}{\partial \vec{n}}\right|_{\partial B}=0 .
$$

There are some existing numerical methods for solving the above nonlinear type PDEs, for instance, time marching [41], lagged diffusivity fixed point (FP) schemes [47] and primal-dual methods [12]. Due to the robustness and simplicity of implementation of the fixed point algorithm, we apply it to solve (B.1)-(B.2) in this work. The idea of the FP method is to first linearize the nonlinear PDE (B.1) by lagging the diffusive coefficient $\frac{\left(1+\sqrt{\gamma_{1}}\right)}{\sqrt{\gamma_{1}+|\nabla f|^{2}}}$ and the relevant parts of right-hand side of (B.1) (like $\Psi_{\beta, \gamma_{2}}^{f}(b)$ and $e^{-\left[\left|f(b)-g\left(b^{\prime}\right)\right|^{2}+\gamma_{2}\left|b-b^{\prime}\right|^{2}\right] / \beta}$ ) by one iteration, and then apply the fixed point method to solve linear problem for $f$. More precisely, the FP iterative method is described as follows:

- Assume we have $f^{n}$.

- Solve for $f^{n+1}$

$$
\begin{aligned}
& -\operatorname{div}\left(\frac{\left(1+\sqrt{\gamma_{1}}\right) \nabla f^{n+1}}{\sqrt{\gamma_{1}+\left|\nabla f^{n}\right|^{2}}}\right)+\frac{2 \alpha \Psi_{\beta, \gamma_{2}}^{f^{n}}(b)}{\int_{B} \Psi_{\beta, \gamma_{2}}^{f^{n}}(b) d b} f^{n+1}(b) \\
& -\frac{2 \alpha\left(\Psi_{\beta, \gamma_{2}}^{f^{n}}(b)\right)^{2}}{\int_{B} \Psi_{\beta, \gamma_{2}}^{f^{n}}(b) d b} \\
& \quad \times \int_{B} g\left(b^{\prime}\right) e^{-\left[\left|f^{n}(b)-g\left(b^{\prime}\right)\right|^{2}+\gamma_{2}\left|b-b^{\prime}\right|^{2}\right] / \beta} d b^{\prime}=0 .
\end{aligned}
$$

The following discretization process of (B.1)-(B.2) is fully inspired by [10]. We introduce the lattice of coordinates $\left(i h_{y}, j h_{x}\right)$, where

$$
\begin{aligned}
& h_{y}=\frac{1}{M-1}, \quad h_{x}=\frac{1}{N-1}, \\
& 0 \leq i \leq M-1, \quad 0 \leq j \leq N-1 .
\end{aligned}
$$

We denote by $f_{i, j}^{n}$ the $n$th FP iteration of $f$ at point $\left(i h_{y}, j h_{x}\right)$ and by $a_{i, j}^{n} \quad$ an approximation of $\frac{1+\sqrt{\gamma_{1}}}{\sqrt{\gamma_{1}+\left|\nabla f^{n}\left(i h_{y}, j h_{x}\right)\right|^{2}}}$. Then we discretize $\frac{\left(1+\sqrt{\gamma_{1}}\right) \partial_{x} f}{\sqrt{\gamma_{1}+|\nabla f|^{2}}}$ by 


$$
\begin{aligned}
& a_{i, j}^{n} \partial_{x} f^{n+1}\left(i h_{y}, j h_{x}\right) \text { and } \partial_{x}\left(\frac{\left(1+\sqrt{\gamma_{1}}\right) \partial_{x} f}{\sqrt{\gamma_{1}+|\nabla f|^{2}}}\right) \text { by } \\
& \frac{1}{2 h_{x}^{2}}\left[\left(a_{i, j-1}^{n}+a_{i, j}^{n}\right) f_{i, j-1}^{n+1}-\left(a_{i, j-1}^{n}+2 a_{i, j}^{n}+a_{i, j+1}^{n}\right) f_{i, j}^{n+1}\right. \\
& \left.\quad+\left(a_{i, j}^{n}+a_{i, j+1}^{n}\right) f_{i, j+1}^{n+1}\right] .
\end{aligned}
$$

In the same way we discretize $\frac{\left(1+\sqrt{\gamma_{1}}\right) \partial_{y} f}{\sqrt{\gamma_{1}+|\nabla f|^{2}}}$ by $a_{i, j}^{n} \partial_{y} f^{n+1} \times$ $\left(i h_{y}, j h_{x}\right)$ and $\partial_{y}\left(\frac{\left(1+\sqrt{\gamma_{1}}\right) \partial_{y} f}{\sqrt{\gamma_{1}+|\nabla f|^{2}}}\right)$ by

$$
\begin{aligned}
& \frac{1}{2 h_{y}^{2}}\left[\left(a_{i-1, j}^{n}+a_{i, j}^{n}\right) f_{i-1, j}^{n+1}-\left(a_{i-1, j}^{n}+2 a_{i, j}^{n}+a_{i+1, j}^{n}\right) f_{i, j}^{n+1}\right. \\
& \left.\quad+\left(a_{i, j}^{n}+a_{i+1, j}^{n}\right) f_{i+1, j}^{n+1}\right] .
\end{aligned}
$$

We also denote by $b_{i, j}^{n}$ an approximation of $\frac{\Psi_{\beta, \gamma_{2}}^{f^{n}}\left(i h_{y}, j h_{x}\right)}{\int_{B} \Psi_{\beta, \gamma_{2}}^{f^{n}}(b) d b}$ and by $c_{i, j}^{n}$ an approximation of

$$
\begin{aligned}
& \frac{\left(\Psi_{\beta, \gamma_{2}}^{f^{n}}\left(i h_{y}, j h_{x}\right)\right)^{2}}{\int_{B} \Psi_{\beta, \gamma_{2}}^{f^{n}}(b) d b} \\
& \quad \times \int_{B} g\left(b^{\prime}\right) e^{-\left[\left|f^{n}\left(i h_{y}, j h_{x}\right)-g\left(b^{\prime}\right)\right|^{2}+\gamma_{2}\left|\left(i h_{y}, j h_{x}\right)-b^{\prime}\right|^{2}\right] / \beta} d b^{\prime} .
\end{aligned}
$$

The last approximations imply computation of definite integrals. Due to its integrands being the piecewise constant functions, its computation is the summation of appropriate function values on lattice grid multiplied by the value of grid element. For example, for $\int_{B} \Psi_{\beta, \gamma_{2}}^{f}(b) d b$ we have

$$
\begin{aligned}
\int_{B} \Psi_{\beta, \gamma_{2}}^{f}(b) d b= & \frac{1}{(M-1) \cdot(N-1)} \\
& \times \sum_{i=0}^{M-1} \sum_{j=0}^{N-1} \Psi_{\beta, \gamma_{2}}^{f}\left(i h_{y}, j h_{x}\right) .
\end{aligned}
$$

Finally, we obtain the following semi-implicit scheme

$$
\begin{aligned}
& -\frac{1}{2 h_{y}^{2}}\left(a_{i-1, j}^{n}+a_{i, j}^{n}\right) f_{i-1, j}^{n+1}-\frac{1}{2 h_{x}^{2}}\left(a_{i, j-1}^{n}+a_{i, j}^{n}\right) f_{i, j-1}^{n+1} \\
& +\left[\frac{1}{2 h_{y}^{2}}\left(a_{i-1, j}^{n}+2 a_{i, j}^{n}+a_{i+1, j}^{n}\right)\right. \\
& \left.+\frac{1}{2 h_{x}^{2}}\left(a_{i, j-1}^{n}+2 a_{i, j}^{n}+a_{i, j+1}^{n}\right)+2 \alpha \cdot b_{i, j}^{n}\right] f_{i, j}^{n+1} \\
& -\frac{1}{2 h_{x}^{2}}\left(a_{i, j}^{n}+a_{i, j+1}^{n}\right) f_{i, j+1}^{n+1} \\
& -\frac{1}{2 h_{y}^{2}}\left(a_{i, j}^{n}+a_{i+1, j}^{n}\right) f_{i+1, j}^{n+1}=2 \alpha \cdot c_{i, j}^{n},
\end{aligned}
$$

where

$$
\begin{gathered}
f_{i, j}^{0}=g\left(i h_{y}, j h_{x}\right) \quad \text { for } 0 \leq i \leq M-1,0 \leq j \leq N-1, \\
f_{0, j}^{n}=f_{1, j}^{n}, \quad f_{M-1, j}^{n}=f_{M-2, j}^{n} \quad \text { for } 0 \leq j \leq N-1, \\
f_{i, 0}^{n}=f_{i, 1}^{n}, \quad f_{i, N-1}^{n}=f_{i, N-2}^{n} \quad \text { for } 0 \leq i \leq M-1 .
\end{gathered}
$$

Now the discrete problem can be written in the matrix-vector form as $M N \times M N$ linear system

$A\left(f^{n}\right) f^{n+1}=b\left(f^{n}\right)$

where $A\left(f^{n}\right)$ is block tridiagonal, symmetric and positive definite (SPD).

In order to solve (B.3), one may apply a variety of iterative methods, like preconditioned conjugate gradients (PCG) methods (see [21]) and multi-grid methods (see [33]). In this work we don't deal with such kind of problem, thus we use here the standard MATLAB function to solve the above linear system for each iteration. The final output, which is the grey-level image, is the desired segmentation. In order to present the results in a pleasant way, the segmentation contour should be obtained. To achieve this, we threshold the accepted segmentation image by graythresh MATLAB function using Otsu's method [37], which chooses the threshold to minimize the intra-class variance of the black and white pixels, and covert it to the binary image. Finally, Canny edge detector [7] is applied to the binary image, and achieved segmentation contour is applied to the original image $g$.

\section{References}

1. Adam, D., Hareuveni, O., Sideman, S.: Semiautomatic border tracking of cine echocardiographic ventricular images. IEEE Trans. Med. Imaging 6, 266-271 (1987)

2. Bardinet, E., Cohen, L.D., Ayache, N.: Tracking medical 3D data with a deformable parametric model. In: Proc. 3rd Eur. Conf. Computer Vision (ECCV'96), Cambridge, UK (1996)

3. Billingsley, P.: Convergence of Probability Measures. Wiley Series in Probability and Mathematical Statistics. Wiley, New York (1968)

4. Brook, A., Kimmel, R., Sochen, N.: Geometry motivated variational segmentation for color images. In: Proc. Scale Space 2001, Vancouver, Canada. LNCS, vol. 2106, pp. 362-370. Springer, Berlin (2001)

5. Brook, A., Kimmel, R., Sochen, N.: Variational restoration and edge detection for color images. J. Math. Imaging Vis. 18(3), 247 268 (2003)

6. Burt, P.J., Adelson, E.H.: The Laplacian pyramid as a compact image code. IEEE Trans. Commun. 31, 532-540 (1983)

7. Canny, J.: A computational approach to edge detection. IEEE Trans. PAMI 8(6), 679-698 (1986)

8. Caselles, V., Catte, F., Coll, T., Dibos, F.: A geometric model for active contours. Numer. Math. 66, 1-31 (1993)

9. Caselles, V., Kimmel, R., Sapiro, G.: Geodesic active contours. Int. J. Comput. Vis. 22(1), 61-79 (1997)

10. Catte, F., Lions, P.L., Morel, J.M., Coll, T.: Image selective smoothing and edge detection by nonlinear diffusion. SIAM J. Numer. Anal. 29(1), 182-193 (1992) 
11. Chakraborty, A., Satib, L.H., Duncan, J.S.: Deformable boundary finding in medical images by integrating gradient and region information. IEEE Trans. Med. Imaging 15, 859-870 (1996)

12. Chan, T., Golub, G., Mulet, P.: A non-linear primal-dual method for TV-based image restoration. In: Lecture Notes in Control and Information Sciences, vol. 219, pp. 241-252 (1996)

13. Chan, T., Sandberg, B., Vese, L.: Active contours without edges for vector-valued images. J. Vis. Commun. Image Represent. 11(2), 130-141 (2000)

14. Chan, T., Vese, L.: Active contours without edges. IEEE Trans. Image Process. 10(2), 266-277 (2001)

15. Choy, M.M., Jin, J.S.: Extracting endocardial borders from sequential echocardiographic images using mathematical morphology and temporal information to improve contour accuracy. IEEE Eng. Med. Biol. Mag. 116-121 (1998)

16. Cohen, L.D.: On active contour models and balloons. CVGIP: Image Underst. 53(2), 211-218 (1991)

17. Dierkes, U., Hildebrandt, S., Kuster, A., Wohlrab, O.: Minimal Surfaces I. Springer Ser., vol. 295 (1991)

18. Evans, L.C.: Partial Differential Equations. Graduate Studies in Mathematics, vol. 19 (1998)

19. Gilbarg, D., Trudinger, N.S.: Elliptic Partial Differential Equations of Second Order. A Series of Comprehensive Studies in Mathematics, vol. 234 (1998)

20. Goldenberg, R., Kimmel, R., Rivlin, E., Rudzsky, M.: Fast geodesic active contours. IEEE Trans. Image Process. 10(10), 14671475 (2001)

21. Golub, G.H., Van Loan, C.F.: Matrix Computations, 2nd edn. The John Hopkins University Press, Baltimore (1989)

22. Imura, T., Yamamoto, K., Kanamori, K., Mikami, T., Yasuda, H.: Non-invasive ultrasonic measurement of the elastic properties of the human abdominal aorta. Cardiovasc. Res. 20, 208-214 (1986)

23. Ip, H.H.S., Hanka, R., Hongying, T.: Segmentation of the aorta using temporal active contour modelwith regularization scheduling. In: Proc. Int. Soc. Opt. Eng., vol. 3034, pp. 323-332 (1997)

24. Kass, M., Witkin, A., Terzopoulos, D.: Snakes: Active contour models. Int. J. Comput. Vis. 1, 321-331 (1988)

25. Kimmel, R.: Fast edge integration. In: Osher, S., Paragios, N. (eds.) Geometric Level Set Methods in Imaging, Vision and Graphics, pp. 59-78. Springer, Berlin (2003)

26. Kimmel, R., Malladi, R., Sochen, N.: On the geometry of texture. Report LBNL 39640, LBNL, UC-405 UC Berkeley, November 1996

27. Kimmel, R., Sochen, N.: Geometric-variational approach for color image enhancement and segmentation. In: Lecture Notes in Computer Science, vol. 49, pp. 294-305. Springer, Berlin (1999)

28. Kluzner, V., Wolansky, G., Zeevi, Y.Y.: Minimal surfaces, measure-based metric and image segmentation. CCIT Report No. 605, EE Pub No. 1562, November 2006

29. Kluzner, V., Wolansky, G., Zeevi, Y.Y.: A geometric-functionalbased image segmentation and inpainting. In: Proc. SSVM 2007, First International Conference, Ischia, Italy. LNCS, vol. 4485, pp. 165-177. Springer, Berlin (2007)

30. Kluzner, V., Wolansky, G., Zeevi, Y.Y.: Geometric approach to image inpainting. J. Math. Imaging Vis. (2008, submitted)

31. Kovacevic, D., Loncaric, S., Sorantin, E.: Deformable contour based method for medical image segmentation. In: Proc. 21st Int. Conf. Information Technology Interfaces (ITI'99), Zagreb, Croatia, pp. 145-150 (1999)

32. Lieb, E.H., Loss, M.: Analysis. Graduate Studies in Mathematics, vol. 14 (1997)

33. McCormick, S.F. (ed.): Multigrid Methods. Society for Industrial and Applied Mathematics, Philadelphia (1987)

34. Morrey, C.B.: The problem of Plateau on a Riemannian manifold. Ann. Math. 49(2), 807-851 (1948)

35. Morel, J.M., Solimini, S.: Variational Methods in Image Segmentation. Birkhauser, Boston (1995)
36. Mumford, D., Shah, J.: Optimal approximations by piecewise smooth functions and associated variational problems. Commun. Pure Appl. Math. 42, 577-685 (1989)

37. Otsu, N.: A threshold selection method from gray-level histograms. IEEE Trans. Syst. Man Cybern. 9(1), 62-66 (1979)

38. Perona, P., Malik, J.: Scale-space and edge detection using anisotropic diffusion. IEEE Trans. PAMI 12(7), 629-638 (1990)

39. Polyakov, A.M.: Quantum geometry of bosonic strings. Phys. Lett. B 103, 207-210 (1981)

40. Ravhon, R., Adam, D., Zelmanovitch, L.: Validation of ultrasonic image boundary recognition in abdominal aortic aneurysm. IEEE Trans. Med. Image 20(8), 751-763 (2001)

41. Rudin, L.I., Osher, S., Fatemi, E.: Nonlinear total variation based noise removal algorithms. Physica D 60, 259-268 (1992)

42. Sagiv, C., Sochen, N.A., Zeevi, Y.Y.: Gabor-space geodesic active contours. In: Zommer, G., Zeevi, Y.Y. (eds.) Algebraic Frames for Perception-Action Cycle. Lecture Notes in Computer Science, vol. 1888, pp. 309-318. Springer, Berlin (2000)

43. Sagiv, C., Sochen, N.A., Zeevi, Y.Y.: Integrated active contours for texture segmentation. IEEE Trans. Image Process. 1(1), 1-19 (2004)

44. Sochen, N.A., Kimmel, R., Malladi, R.: A general framework for low-level vision. IEEE Trans. Image Process. 7, 310-318 (1998)

45. Sochen, N.A., Kimmel, R., Malladi, R.: From high energy physics to low-level vision. Report LBNL 39243, LBNL, UC Berkeley, CA 94720, August 1996

46. Sochen, N.A., Zeevi, Y.Y.: Representation of colored images by manifolds embedded in higher dimensional non-Euclidean space. In: Proc. IEEE ICIP'98, Chicago (1998)

47. Vogel, C., Oman, M.: Iterative methods for total variation denoising. SIAM J. Sci. Stat. Comput. 17(1), 227-238 (1996)

48. Wohlrab, O.: Zur numerischen Behandlung von parametrischen Minimalflachen mit halbfreien Randern. Dissertation, Bonn (1985)

49. You, Y.L., Kaveh, M.: A regularization approach to joint blur identification and image restoration. IEEE Trans. Image Process. 5(3), 416-428 (1996)

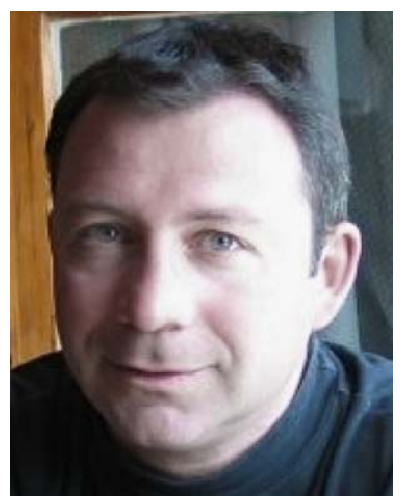

Vladimir Kluzner is a Research Staff Member at the Document Processing and Management Group, IBM Haifa Research Lab, IBM Corporation. Currently his efforts are concentrated in large scale digitization project transforming the printed heritage (ancient books) into digitally available resources. He has received his B.Sc., M.Sc. and Ph.D. in Applied Mathematics from the Technion-Israel Institute of Technology in 1992, 1999 and 2007 respectively. He has worked as an image processing researcher in El-Op ElectroOptics Industries (subsidiary of Elbit Systems), Israel in 1999-2001, being involved in developing new algorithms for the next generation of various military systems, conducting the research in the areas of electronic stabilization, optical distortions correction and motion detection. He has also worked as an algorithm developer in UltraSPECT, Israel in 2005-2006, being involved in developing new algorithms for the next generation of Wide Beam Reconstruction (WBR) methods in SPECT imaging. His main research interests contain Numerical Methods in Linear algebra, Variational Calculus, Computer Vision, Image Processing, Computerized Tomography, OCR Techniques and Document Processing. 


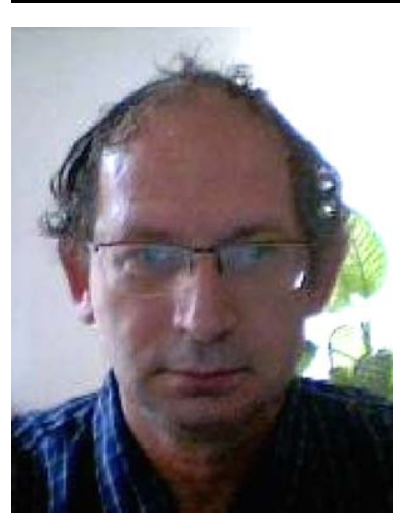

Gershon Wolansky graduated from the Hebrew University at Jerusalem and received his Ph.D. degree in mathematics at the Courant Institute, NY, USA, in 1985 . He is currently a professor in the department of mathematics at the Technion, Israel Institute of technology. His fields of interest contain variational calculus, optimal mass transport (Monge-Kantorovich), computer vision, mathematical biology (chemotaxis), optics, and related fields such as dynamical systems, stochastic dynamics, and elliptic/parabolic differential equations. He received a postdoctoral scholarship from the Wolf Foundation in 1987, the Sara Leedy award in mathematics in 1994 and 1995, GFAT Academic Lectureship, France, the Sanford Kaplan Creative Management prize, and the $\mathrm{H}$. Rich Innovation prize in 2000. Professor Wolansky had 6 graduate students, 2 postgraduate, and he published more than 50 papers in international journals. He is also working as a counselor for several technological companies and has 7 registered patents in optics and ophthalmology.

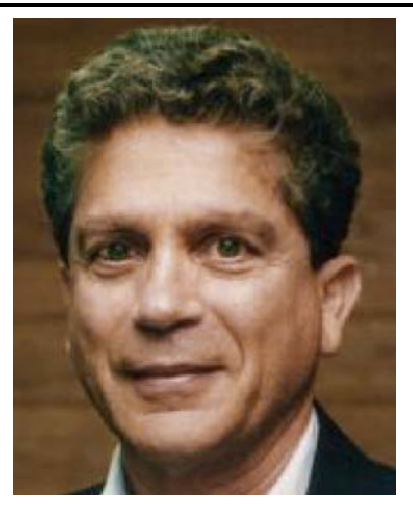

Yehoshua Y. Zeevi is the Barbara and Norman Seiden Professor of Computer Sciences in the department of Electrical Engineering, Technion-Israel Institute of Technology. He is the Director of the Ollendorff Center for Vision and Image Sciences, and the Head of the Zisapel Center for NanoElectronics. He received his Ph.D. from the University of California, Berkeley, and was subsequently a Vinton Hayes Fellow at Harvard University, where he had been a regular visitor for many years. He was also a Visiting Professor at MIT, the CAIP Center of Rutgers University, and Columbia University. His major research has been devoted to vision and image sciences, and to signal and image representation and processing. He is a Fellow of the SPIE and the Rodin Academy, the Editor-in-Chief of the Journal of Visual Communication and Image Representation, published by Elsevier. $\mathrm{He}$ is one of the founders of iSight, Inc.- - a company devoted to digital photography and real time image processing, and of UltraGuidea company that developed innovative computerized systems for visual guidance in ultrasound-assisted medical procedures, and of Corticaa company that developed new large scale systems for tracking and deep content classification. He is a member of the IEEE Committee for Multidimensional Signal processing and of the Board of the Technion. 